\title{
Les ports, havres et rivières navigables de Normandie dans l'enquête des amirautés de 1665
}

Normandy's Ports, Harbours and Navigable Rivers in the Survey of the Admiralties of 1665

Édouard Delobette

\section{OpenEdition}

\section{Journals}

Édition électronique

URL : http://journals.openedition.org/abpo/2867

DOI : 10.4000/abpo.2867

ISBN : $978-2-7535-3977-8$

ISSN : 2108-6443

\section{Éditeur}

Presses universitaires de Rennes

\section{Édition imprimée}

Date de publication : 15 décembre 2014

Pagination : $39-80$

ISBN : 978-2-7535-3975-4

ISSN : 0399-0826

\section{Référence électronique}

Édouard Delobette, "Les ports, havres et rivières navigables de Normandie dans l'enquête des amirautés de 1665 », Annales de Bretagne et des Pays de l'Ouest [En ligne], 121-4 | 2014, mis en ligne le 15 décembre 2016, consulté le 19 avril 2019. URL : http://journals.openedition.org/abpo/2867 ; DOI : $10.4000 / a b p o .2867$ 


\title{
Les ports, havres et rivières navigables de Normandie dans l'enquête des amirautés de 1665
}

\author{
Édouard DELOBETTE \\ Chercheur associé au CRHQ/CNRS, UMR 6583, Université de Caen
}

«Beaulx amys, puys que surgir ne povons à bon port, mettons nous à la rade, je ne scay où. "

Rabelais, Le Quart Livre des faicts et dicts hérö̈ques du bon Pantagruel, 1552.

Entré au service de "l'État Mazarin " (Daniel Dessert), Jean-Baptiste Colbert est convaincu dès 1650 que " pour remettre le commerce, il y a deux choses nécessaires : la sûreté et la liberté ", mais à la condition préalable d'une « militarisation accrue » du littoral par l'État ${ }^{1}$. S'inspirant des projets politiques du cardinal de Richelieu et du surintendant Fouquet, Colbert présente au roi en août 1664 son " grand dessein " censé permettre à la France de parvenir notamment à la suprématie militaire et navale pour « la gloire du roi et le bien de l'État ${ }^{2}{ }$. Ce grand projet comprend la construction

1. LE BOUËDEC, Gérard et LLINARES, Sylviane, « Le port comme lieu de conflit d'autorité (XVI$\mathrm{XIX}^{\mathrm{e}}$ siècles) ", Les Cahiers du CRHQ, n ${ }^{\circ}$ 1, URL : [http://www.crhq.cnrs.fr/cahiers/page-cahier. php?id_num=6]; mis en ligne le 5 janvier 2009, consulté le 14 septembre 2013. « Le xvII siècle constitue un tournant. Le nouveau discours sur le littoral est sans ambiguïté : l'État doit le contrôler car il est en fait un espace stratégique comme frontière et comme point d'appui de sa politique de projection océanique, qu'elle soit militaire ou marchande. Il en résulte une militarisation accrue du littoral, certes à travers les fortifications, mais, et c'est nouveau, avec de grands équipements militaires portuaires. D'ailleurs, le choix se porte sur des sites en eau profonde pour limiter les aménagements. Leur double dimension, portuaire et industrielle pour la construction de flottes spécialisées de gros tonnages, en fait des gros consommateurs d'espaces littoraux d'autant que ces créations parfois ex nihilo s'accompagnent de créations urbaines ". Citation à rapprocher de EvELYN, John, Navigation and Commerce, their origin and progress, Londres, ed. J.R., 1674, p. 15-17 et 32-33 : « To pretend to universal monarchy without fleets was long since looked upon, as a political chimaera [...] whoever commands the ocean, commands the trade of the world, and whoever commands the trade of the world, commands the riches of the world, and whoever is master of that, commands the world itself. "

2. JACQUART, Jean, "Colbert ", dans MÉChoulan, Henry, CoRnetTe, Joël (dir.), L'État Classique 1652-1715. Regards sur la Pensée Politique de la France dans le Second XVIT siècle, Paris, Vrin, 1996, p. 189-190. 
d'une flotte de guerre, la reconstitution de la marine marchande, le contrôle administratif et social des gens de mer $^{3}$. Pour atteindre ce but, l'État doit nécessairement acquérir la connaissance la plus étendue et détaillée possible du potentiel portuaire du royaume par des enquêtes selon le modèle des Instructions aux maîtres des requêtes, aussi appelées le " grand questionnaire ${ }^{4}$ ".

Ainsi, l'enquête menée en 1665 dans les ressorts des amirautés de France sur le lestage et délestage des navires marchands montre que la monarchie prend désormais en haute considération la problématique des accès nautiques des rivières, ports, havres et rades du royaume ${ }^{5}$. L'enquête ne néglige pas non plus les petits ports et havres, indispensables relais et points d'appui des activités maritimes et littorales car "c'est en initiant des recherches sur les navigations intermédiaires entre les grands ports (cabotage) et sur les petites pêches que sont apparus ces petits ports comme essentiels à la compréhension de l'organisation des littoraux ${ }^{6}$ ". C'est ce que l'on entend interpréter ici à travers une approche consacrée à la Normandie maritime.

Les principales sources de cette étude proviennent des procès-verbaux reçus des amirautés normandes, croisés avec d'autres enquêtes, mémoires ou correspondances administratives reçues par Colbert ${ }^{7}$. Par le recours à une forme de récit tantôt factuelle, tantôt par l'assemblage de témoignages successifs suivant une ligne de force conductrice ou " emplotment ${ }^{8}$ " (Paul Ricœur), l'article s'organise dans un premier temps avec l'analyse du document SH 48, présente ensuite les caractéristiques naturelles des ports de

3. LE BouËDEc, Gérard et CHAPPÉ, François (dir.), Pouvoirs et littoraux du XV $v^{e}$ au XXe siècle, Actes du colloque international de Lorient, 24-26 septembre 1998, Rennes, PUR/UBS, 2000.

4. Meyer, Jean, Colbert, Paris, Hachette, 1981, p. 223. LEBRun, François, "Les grandes enquêtes statistiques des XVII ${ }^{\mathrm{e}}$ et $\mathrm{XVIII}^{\mathrm{e}}$ siècles sur la généralité de Tours (Maine, Anjou, Touraine) ", Annales de Bretagne, t. 72, n² 2, 1965, p. 338-339.

5. BochacA, Michel, SARrazin, Jean-Luc (dir.), Ports et littoraux de l'Europe atlantique : Transformations naturelles et aménagements humains (XIV-XVte siècles), PUR, 2007. WAUTERS, Éric (dir.), Les ports normands : un modèle?, Actes colloque Rouen-Le Havre, 28-29 mai 1998, Presses universitaires Rouen-Le Havre, 1999. Journées d'études de la Société française d'histoire urbaine et du Centre de recherche en histoire quantitative (CRHQ), UMR 6583/CNRS/CRHQ-UCBN, Les zones portuaires : aménagements, réaménagements, réhabilitation de l'Antiquité à nos jours, Caen, 16-17 janvier 2014.

6. LE BouËDEC, Gérard, "Les petits ports bretons du XVI ${ }^{\mathrm{e}}$ au XIX ${ }^{\mathrm{e}}$ siècle ", Rives Méditerranéennes, $\mathrm{n}^{\circ}$ 35, 2010, p. 61 .

7. Les mémoires administratifs concernant les ports et littoraux de Basse-Normandie au XVII ${ }^{\mathrm{e}}$ siècle, moins nombreux que pour la Haute-Normandie, sont complétés avec : GouHIER, Pierre, L'Intendance de Caen en 1700, Paris, Comité des travaux historiques et scientifiques (CTHS), 1998; MEYER, Jean, "Un exemple à méditer : les Cahiers des Annales de Normandie ", Annales de Bretagne, t. 72, n² 2, 1965, p. 347-348.

8. White, Hayden V., Metahistory. The Historical Imagination in Nineteenth-Century Europe, Baltimore, The John Hopkins University press, 1975, p. 29, relève trois formes majeures de structuration du récit historique : par l'argument factuel, par le discours idéologique, ou par "l'emplotment " de Paul Ricœur, soit une succession d'histoires raccordées à une même intrigue ou thématique. 
Normandie, puis dresse l'état des lieux des structures portuaires et littorales de la province vers $1665^{9}$.

\section{Le document SH 48 et la procédure d'enquête}

\section{Les instructions officielles}

L'enquête des amirautés de 1665 est actuellement conservée au Service historique de la Défense de Vincennes, fonds Marine, en cote SH $48^{10}$. En raison du caractère encore embryonnaire des correspondances reçues des commissaires et intendants de marine avant 1669, le document $\mathrm{SH} 48$, rarement utilisé jusqu'à présent à l'exception du mémoire sur la généralité de Rouen présenté par Edmond Esmonin ${ }^{11}$, s'avère être d'un très grand intérêt informatif dans une période charnière de la vie maritime et portuaire du royaume.

L'organisation interne du recueil SH 48, que l'on appellera désormais le SH 48 par commodité, débute par une table des matières suivie d'un exemplaire imprimé de l'arrêt du Conseil d'en haut du 24 janvier 1665, signé par Hugues de Lionne, officiellement en charge des affaires de la Marine ( $\mathrm{f}^{\circ} 1$ et suiv.). L'arrêt est complété par l'instruction manuscrite de JeanBaptiste Colbert, conseiller du roi en ses conseils, intendant des finances, exerçant à titre officieux depuis 1661 la direction du département de la Marine et des fortifications littorales. Cette instruction est intitulée "Pour le commerce " et présentée sous la forme d'un questionnaire ( $f^{\circ} 5$ et suivants). Les exemplaires de ces deux pièces sont ensuite hâtivement expédiés dans le courant du mois à tous les procureurs du roi des sièges particuliers d'amirauté du royaume, y compris aux juridictions de Bretagne qui connaissent des causes maritimes et littorales ${ }^{12}$. Le reste du SH 48 collationne, depuis le folio 9 jusqu'au folio 502, les procès-verbaux reçus de la plupart des sièges d'amirauté, dont le sens du classement géographique nous échappe (voir tableau ci-dessous).

L'arrêt du 24 janvier 1665, donné en présence du roi, est motivé par diverses plaintes adressées au Conseil sur l'état de certains ports, rades, havres, baies, rivières diverses qui seraient devenus de jour en jour moins sûrs et moins praticables aux navires. Le trafic fluvial et maritime et le mouvement des navires de guerre pourraient en être durablement affectés. D'après l'exposé de l'arrêt, les responsables en seraient les capitaines,

9. Le point de réflexion initial provient de LEMARCHAND, Guy, " Problématique pour une étude des ports de Normandie ", dans WAUTERS, Éric (dir.), Les ports normands..., op. cit., p. 18-19.

10. Le sigle SH signifie Service Hydrographique. L'ancienne cote de référence du SH 48 était $m s n^{\circ} 271$.

11. Voysin de La Noiraye, Jean-Baptiste, Mémoire sur la Généralité de Rouen (1665), (EsMONIn, Edmond, éd.), Paris, Hachette, 1913.

12. LE BouËDEC, Gérard (éd.), L'Amirauté en Bretagne. Des origines à la fin du XVIII siècle, PUR, 2012 (présentation de la thèse de Joachim Darsel [1954]). 


\section{Tableau 1 - Les origines géographiques des procès-verbaux des amirautés}

\begin{tabular}{|c|c|}
\hline $\begin{array}{l}\text { Procès-verbaux reçus des amirautés (avec } \\
\text { parfois leurs ports " obliques " ou secon- } \\
\text { daires) ou autres juridictions (ordre de } \\
\text { classement du SH 48). }\end{array}$ & $\begin{array}{l}\text { Amirautés ou autres juridictions dont les } \\
\text { procès-verbaux font défaut dans le SH } 48 \\
\text { (le classement des sièges reprend celui } \\
\text { dressé par les commis). }\end{array}$ \\
\hline $\begin{array}{l}\text { Dunkerque, Gravelines, Boulogne, St } \\
\text { Valery, Bourg d'Ault, Eu et Treport, } \\
\text { Sables d'Ollone, Roüen, Dieppe, St-Valery } \\
\text { en Caux, Havre de grace, Caudebec, } \\
\text { Honfleur, Estreham, Caen, Bayeux port } \\
\text { et annelles, Carentan, Grand Camp, } \\
\text { La hogue et Geneville [Quinéville], } \\
\text { Barfleur, Cherbourg, Coustances, } \\
\text { Quimper Corentin et Audierne, Bordeaux, } \\
\text { Bayonne, Costes et isles de Xaintonges, } \\
\text { La Rochelle, Marseille, Toulon, Arles, } \\
\text { Martigues, St Tropez, Narbonne, Cap } \\
\text { de Cette, Agde, Frontignan, Serignan, } \\
\text { Vendres, St Malo et Cancale, Morlaix, Ruiz, } \\
\text { Vannes, Auray, Hennebon, Quimperlé, } \\
\text { Treguier, Cornuaille, Guerrande. }\end{array}$ & $\begin{array}{l}\text { Calais, Abbeville et le Crotoy, Toucques, } \\
\text { Dives, Portbail et Carteret, Grandville } \\
\text { et Genet, Brouage et isles, Antibes, } \\
\text { Aiguemorte, St Brieuc, Lanion, Brest St } \\
\text { Renan et le Conquet, Lesnevan et Roscoff, } \\
\text { Chateaulin et Landerneau, Concarneau, } \\
\text { Pinpol et isles de Bréat, Piriac Le Croisic } \\
\text { et St Nazaire, Belle isle, Nantes. }\end{array}$ \\
\hline
\end{tabular}

Source : SHD Vincennes, cote SH 48. NB : orthographe toponymique d'origine conservée; inversion de classement des procès-verbaux du siège de Saint-Valéryen-Somme avec celui de Saint-Valéry-en-Caux par rapport à la table des matières, due à une confusion de la part des commis. En gras, les amirautés normandes. Les sièges de Fécamp, Collioure-Perpignan (créé en 1691)a , La Ciotat et Fréjus ne figurent nulle part dans le document. En Bretagne, où n'existent pas de sièges d'amirauté avant 1691 , les causes maritimes et littorales sont connues par les juridictions ordinaires (sénéchaussées). L'amirauté de Brouage est remplacée depuis 1645 environ par celle de Marennes. Les sièges d'amirautés coloniales ${ }^{\mathrm{b}}$, établis par l'édit de 1717 et ceux de Corse (1768), ne figurent pas dans le document.

a. Lavoux, Régis, L'amirauté de Collioure (1691-1790), thèse doctorat droit, Perpignan, 1998, dact., 581 p.

b. Aux colonies, le pouvoir de l'Amirauté de France s'établit progressivement avec la guerre de course durant la guerre de la ligue d'Augsbourg (GASSER, Jacques, "Les droits de l'Amirauté de France à la Martinique (1696-1697) ", Chronique d'Histoire maritime, n ${ }^{\circ}$, décembre 2012, p. 27-40).

maîtres et patrons de navires qui, " pour épargner la dépense que cause la décharge du lest, ont conservé la criminelle habitude de jeter leur lest, en tout ou partie, en arrivant dans les ports \& rades " en dépit des ordonnances antérieures visant à faire cesser cet abus ${ }^{13}$. L'arrêt ordonne ainsi aux

13. Valin, René Josué, Nouveau commentaire sur l'ordonnance de la Marine du mois d'août 1681, La Rochelle, Légier imp., 1776, t. II, 1776, p. 509, rappelle l'inefficacité des textes réglementaires successifs peu observés dans les ports et rades (ordonnance de 1629, lettres patentes de janvier 1640, règlement du duc de Vendôme d'octobre 1660 , arrêt du Conseil du 24 janvier 1665). Arch. dép. de Seine-Maritime, 216 BP 371, ordre du 


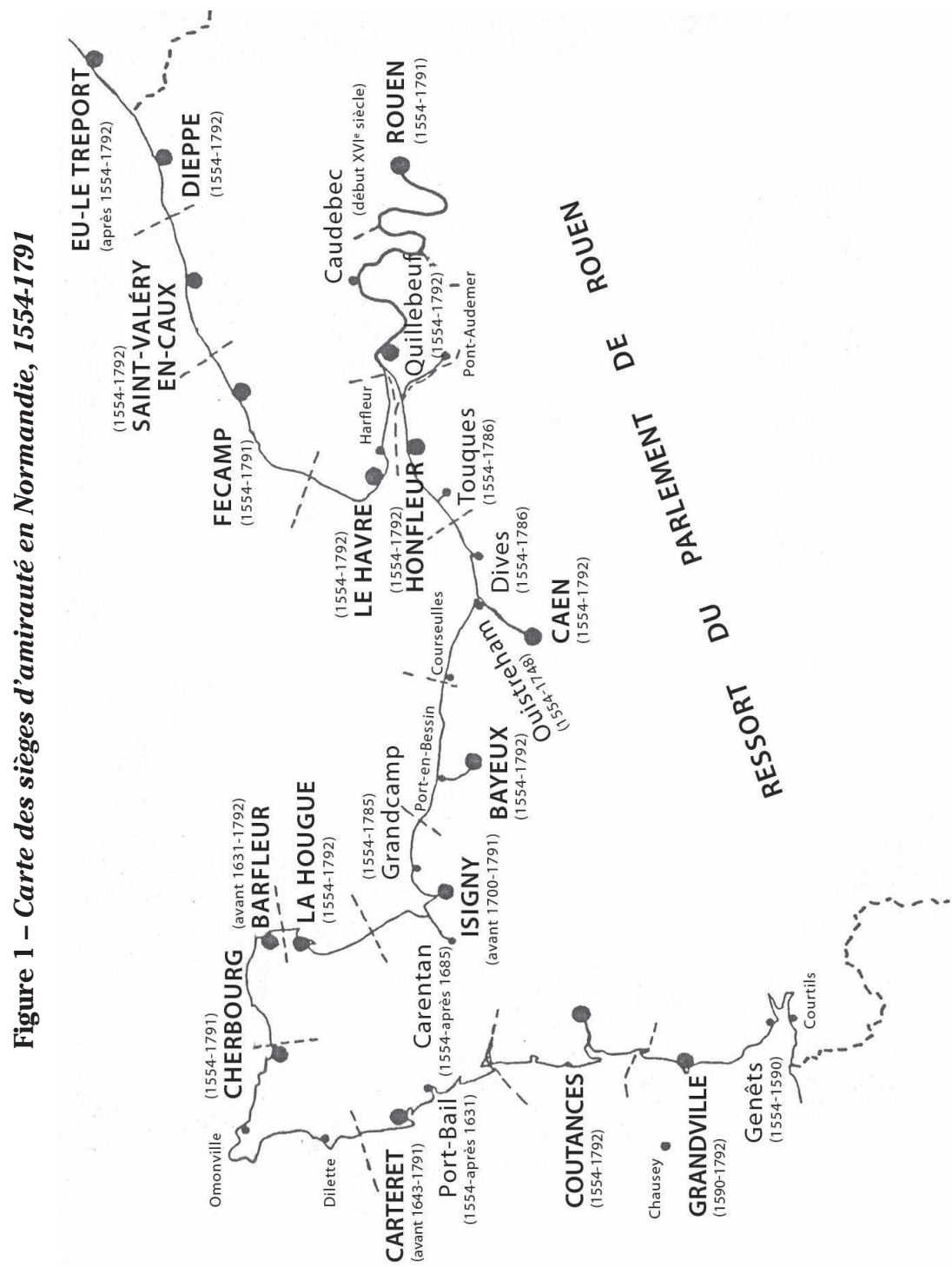

roi, enregistré à l'amirauté du Havre le 26 novembre 1660, faisant défense aux capitaines et maîtres de navires de continuer à jeter le lest de leur navire hors des lieux indiqués " par avidité du gain et pour descharger plus promptement leurs navires au retour de leur voyage ", ce qui finirait par combler les embouchures des ports, havres et rades et provoquerait leur ruine. Duquesne souligne au Havre la responsabilité des capitaines et maîtres étrangers : "L'ancienne rade soubz le Chef de Caux ou de la Hève a esté gastée pour n'y avoir pas eu d'ordre pour empescher le delestage des vaisseaux estrangers qui y attendent d'ordinaire les hautes marées pour monter en Seine. " (BRÉARD, Charles, "Mémoires et documents relatifs aux ports de Normandie : Le Havre, Fécamp, Honfleur ", Bulletin de la Société normande de Géographie, 1892, p. 11.) 
officiers des sièges particuliers d'amirauté et aux autres juges exerçant aux " cours maritimes " de faire la visite et sondage des havres, rades et ports de leurs juridictions et d'enquêter sur leur dépérissement éventuel, l'existence de marques de lieu de décharge des lests, la levée éventuelle de droits sur le lestage et délestage, puis d'envoyer le rapport ou enquête sommaire à Colbert, en vue de la rédaction ultérieure d'un règlement général.

\section{Le port idéal imaginé par Colbert}

L'instruction manuscrite de Colbert, jointe à l'arrêt du Conseil, est rédigée sous la forme d'un questionnaire divisé en 38 articles. Ce document explique scrupuleusement aux officiers des sièges les informations précises que Colbert attend d'eux lorsqu'ils effectueront prochainement la visite de leur juridiction afin de connaître " les bonnes et mauvaises qualitez des ports " ( $f^{\circ} 5$ et suiv.). Contrairement à la méthode habituellement suivie par Colbert ${ }^{14}$, l'urgence du contexte international et maritime explique le caractère hâtif des articles de l'instruction, additionnés sans véritable ordonnancement ni cohésion des thématiques. L'analyse de l'instruction nous apprend surtout comment Colbert, qui reste " un terrien et à beaucoup d'égards un parisien " (Jean Meyer), conçoit théoriquement vers 1665 le port idéal, indispensable à la réalisation de son " grand dessein " sur mer ${ }^{15}$.

Vraisemblablement conseillé par Abraham Duquesne ${ }^{16}$, Colbert définit son modèle portuaire idéal selon trois critères : la topographie, le commerce et la puissance navale. Les articles 1 à 8 et 15 enjoignent aux officiers d'amirauté de rechercher et de décrire les sites littoraux propices à la navigation des vaisseaux, offrant de belles plages et avec des marées

14. MEYER, Jean, Colbert, op. cit., p. 100, 104 et 116-117.

15. Sur les critères d'un bon port retenus en Haute-Normandie énumérés par l'intendant de la généralité de Rouen en 1665 : avoir une rade orientée dans le sud-sud-ouest du port pour faciliter l'entrée des vaisseaux, un faible marnage de moins de 10 pieds d'eau d'écart entre vive eau et morte eau, une profondeur satisfaisante (15-20 pieds d'eau au moins à basse mer de morte eau, avec 8-10 brasses au moins pour le mouillage des vaisseaux), un " plain " de la mer tenant au moins deux heures pour laisser à une flotte le temps d'entrer ou de sortir, des vents correctement orientés pour sortir de la Manche (secteurs nord à est-nord-est et sud-est à est). La rade, bien défendue, doit avoir un bon fond (depuis vase ou terre recherché, à terre-sable moins bon, et de sable encore moins apprécié, fond de roches le plus mauvais) (Esmonin, Edmond, Mémoire..., op. cit., p. 4-5). Les instructions de Colbert s'inspirent en partie de la première édition de 1643 de FouRNIER, Georges (1595-1652), Hydrographie contenant la théorie et la practique de toutes les parties de la navigation..., Paris, Jean Dupuis, 1667, $2^{\mathrm{e}}$ éd., p. 45-55, sur les qualités nécessaires à un bon port, son entretien et la façon de le gâter.

16. De retour de la campagne équivoque de Djidjelli, le chef d'escadre Duquesne séjourne à la cour de janvier à début mai 1665 avant d'entreprendre la visite du littoral normand (VERGÉ-FrancESCHI, Michel, Abraham Duquesne. Huguenot et marin du Roi-Soleil, Paris, éd. France-Empire, 1992, p. 205-209). Le mémoire de Duquesne pour le rétablissement du port du Havre présente de nombreux points communs avec l'instruction de Colbert. (PETER, Jean, Le Port et l'Arsenal du Havre sous Louis XIV, Paris, Economica et ISP, 1995, p. 17). 
réglées et douces, des rades ayant un bon fond de bonne tenue à l'ancrage, sans écueil ni brisants. L'entrée du havre neuf est souhaitée bien ouverte et profonde, d'un accès facile, sans détours (méandres, bancs, etc.) en précisant le tonnage maximum admis pour les vaisseaux en charge ou sur lest, entrant et sortant à toutes heures du jour ou de la nuit sans se soucier de la marée. Le site portuaire doit apparaître net, creux, vaste, assuré contre les vents et les marées. Il doit de plus offrir plusieurs bras et canaux pour recevoir les vaisseaux marchands, ou au moins une rivière pour le transport des marchandises entre le port et l'arrière-pays. Les officiers devront enfin estimer si le site permet le creusement d'un bassin à flot et dresser un devis des travaux éventuels (art. 8 et 36). Ces deux groupes d'articles répondent aux inquiétudes de Colbert, soucieux de savoir si les littoraux français présentent autant d'avantages naturels que ceux du sud de l'Angleterre.

Les articles abordant la question commerciale sont peu nombreux et se cantonnent à la fiscalité. Colbert tient en effet à connaître le détail des taxes, impositions et droits perçus dans chaque port. Outre la lutte menée contre les éventuelles usurpations des droits du roi sur les littoraux commises par des particuliers ou des communautés (art. 35), il redoute aussi les excès d'une fiscalité mal maîtrisée qui détourneraient des trafics marchands français et étrangers vers d'autres lieux, aidés en cela par les " subtilités " du commerce hollandais (art. 16). Fils de grand marchand rémois, Colbert tient enfin à savoir si les diverses juridictions portuaires ou seigneuriales rendent une bonne justice marchande, c'est-à-dire une justice équitable qui ne va pas à l'encontre des intérêts du commerce maritime (art. 17).

Ce sont les questions portant sur les risques maritimes et la sécurité de la navigation qui présentent logiquement le plus grand nombre d'articles dans l'instruction complémentaire de Colbert adressée aux amirautés. Les articles 25 et 26 s'inspirent du modèle hollandais en invitant les officiers à enquêter sur la nécessité de construire des digues ou des môles de protection contre la mer. Les officiers doivent se préoccuper de savoir si le port dispose d'aides à la navigation, un phare ou une tour à l'embouchure (art. 9 et 26), d'un balisage correctement entretenu (art. 27) et s'il y a de bons géographes entretenus sur place (art. 12) ${ }^{17}$. La monarchie admet depuis le $\mathrm{XVI}^{\mathrm{e}}$ siècle la nécessité de mieux connaître les territoires du royaume et ses limites ${ }^{18}$. L'exacte cartographie des côtes comme la lutte contre les dangers

17. «Le Roy veut qu'il soit faict une description exacte de toutes les costes du royaume, et qu'il soit toujours travaillé dans ses ports à dresser des cartes marines sur les rapports et les journaux de ses vaisseaux de guerre " (MEYER, Jean, Colbert, op. cit., p. 126).

18. Konwitz, Josef W., Cartography in France, 1660-1848 : Science, Engineering, and Statecraft, Chicago-Londres, University of Chicago Press, 1987. LiEPPE, Denis, "La représentation cartographique du rivage occidental de la France aux XVII et XVIII siècles ", Communications 1993-1994, Comité de documentation historique de la Marine, Vincennes, Service historique de la Défense, 1995, p. 323-342. RicHARD, Hélène, " Les outils cartographiques à l'usage des marins français à l'époque de Tourville ", dans REFFuvEILLE, Antoine (dir.), Tourville et les marines de son temps, Actes du colloque des Archives départemen- 
maritimes deviennent ainsi un enjeu stratégique majeur ${ }^{19}$. Ce point prend de l'importance au moment où d'autres puissances maritimes comme l'Angleterre édifient des phares aux endroits les plus dangereux du littoral ${ }^{20}$. Rigoult, le procureur du roi de l'amirauté de Saint-Valéry-en-Caux rappelle même ouvertement à Colbert la parfaite connaissance géographique des ports et côtes de France accumulée par la navigation hollandaise ${ }^{21}$.

Cet effort scientifique se poursuit sous le règne de Louis XIV. Le souverain ordonne l'envoi de missions cartographiques composées d'astronomes, d'hydrographes et d'ingénieurs militaires pour dresser les cartes à tracé géométrique des côtes de France ${ }^{22}$. Le besoin de cartes marines plus précises est lié à la formation de coûteuses flottes de combat et de l'expansion du domaine colonial des grandes puissances maritimes. Les jeunes ingénieurs-géographes de la génération de Vauban recourent non seulement à un langage cartographique neuf, bien plus apprécié de Colbert que le système imprécis et suranné de Clerville, tout en se montrant capables d'intégrer dans leurs travaux le contexte de l'art classique propre au règne de Louis XIV. Les officiers d'amirauté doivent aussi mentionner dans leurs procès-verbaux la présence éventuelle de professeurs d'hydrographie pour instruire les pilotes, capables de dresser ou de réviser des cartes marines récentes et d'entretenir les instruments de navigation des bâtiments marchands ou de guerre (art. 12). Ils doivent enfin s'enquérir de l'état des systèmes d'amarrage fiables à quai ou à terre pour les vaisseaux (art. 10). L'instruction reprend et élargit dans les articles 21 et 22 les dispositions de l'arrêt du Conseil portant sur le délestage des navires et l'entreposage du

tales de la Manche, 10-12 sept. 2001, Saint-Lô, Archives départementales de la Manche, 2003, p. 172. Colbert dépêche sur les côtes plusieurs ingénieurs hydrographes : en Baie de Seine, Georges Boissaye du Bocage père réceptionné à l'amirauté du Havre en 1660 (Le Havre, 1626-id., 1696), Sainte-Colombe en Normandie et Saintonge pour dresser des cartes plus précises.

19. Le roi charge en 1676 Seignelay de regrouper les cartes marines et mémoires pour améliorer la sécurité de la navigation : SHD Vincennes, SH 79, Recueil des costes de France, sur l'océan avec les laisses, les bancs, les rochers et les sondes, tels qu'ils paroissent en basse mer, dans les plus grandes marées, par de la Favolière, Samson, Duboccage, le chevalier de Clerville, Levasseur de Beauplan, Sainte-Colombe, Mariette, de La Voye, Tassin et Minet.

20. Les estuaires de la Tyne, de la Humber, les ports et caps de Great Yarmouth, Lowestoft, the Naze, North Foreland, Hastings et Portland Race, Eddystone sont signalés aux navigateurs par des phares équipés de foyers alimentés en charbon de terre. Charles II confie une mission cartographique au capitaine Greenvile Collins pour corriger les nombreuses erreurs et approximations des cartes marines : " [Charles II] who was a great lover of the noble Art of Navigation, finding that there were no sea-charts, or maps of these kingdoms, but what were Dutch, and copies from them, and these very erroneous [...] was pleased in the year 1682 to give me the command of a Yacht for the purpose of making a Survey. " (WILlan, Thomas S., The English Coasting Trade 1600-1750, Manchester University Press, 1938, p. 21-22). Les premiers résultats sont édités en 1693.

21. Bibliothèque nationale de France (désormais BnF), Mélanges Colbert, 128 bis, $\mathrm{f}^{\circ} 773$, $1^{\mathrm{er}}$ mai 1665 .

22. LE BouËDEC, Gérard et CHAPPÉ, François (dir.), Représentations et images du littoral, Actes de la journée d'études de Lorient, 22 mars 1997, PUR, 1998. CoRBIN, Alain, Le Territoire du vide. L'Occident et le désir du rivage, Paris, Flammarion, 1990, p. 226. 
lest à terre ou dans une zone d'immersion réservée, le dragage et aplanissement des fonds des havres de tout lest existant.

Colbert conçoit avec raison qu'un bon port doit nécessairement être bien protégé par un nombre suffisant de soldats (contre les "émotions " ou révoltes urbaines, les attaques ennemies), aptes à repousser des opérations corsaires et de leur donner la chasse (art. 6, 17 et 18) ${ }^{23}$. L'instruction enjoint aux officiers des sièges de faire construire dans les ports des magasins d'armement en vivres, artillerie et munitions navales, puis d'en contrôler la bonne gestion par les gardes-magasins (art. 13 et 37). Leur visite doit aussi s'assurer si l'hinterland peut fournir rapidement par adduction dans des citernes l'eau douce pour les navires (art. 28), les munitions navales (bois, chanvre, fers) et les victuailles (art. 14 et 34) sur la base des informations collectées auprès des capitaines de navire dans les différents ports afin de mieux en répartir les besoins. Un soin tout particulier est donné dans l'instruction à l'information détaillée portant sur les corps de métier et leurs savoirs liés à la construction navale (art. 30), les provenances et qualités des matières premières nécessaires à la " bâtisse " des vaisseaux, notamment les bois de construction, au moment où Colbert met en œuvre la construction d'une flotte de guerre (art. 31).

À plus longue échéance, le " bon port " vu par Colbert suppose la stricte réorganisation utilitariste des rôles et places de chacun dans le volet maritime du " grand dessein ". Les officiers doivent d'abord veiller à faire placer sous leur surveillance ou par d'autres juridictions comme celle des eaux et forêts (art. 17), les catégories sociales qui pourraient entraver l'essor portuaire et naval. Sont clairement désignés par l'instruction les pêcheurs qui embarrassent les accès des ports et rendent illisible le balisage avec leurs apparaux de pêche (art. 27), à nouveau les corsaires (art. 6 et 18), les coupeurs de bois non autorisés qui perturbent les approvisionnements des chantiers navals (art. 32), les gens de mer et travailleurs des ports protestants soupçonnés de vouloir s'exiler (art. 33), les particuliers usurpateurs de droits sur les littoraux (art. 35).

L'instruction annonce également la volonté implicite de Colbert d'introduire un contrôle administratif étroit sur les gens de mer car la création d'une flotte de guerre mobilise des équipages pléthoriques. N'ayant toutefois aucune donnée statistique fiable sur ce point ${ }^{24}$, Colbert s'enquiert par l'article 29 du peuplement de chaque juridiction d'amirauté, ainsi que sa

23. L'obsession de Colbert envers la piraterie remonte au moins à 1650 et s'observe déjà dans le "Mémoire touchant le commerce avec l'Angleterre [1650] ", attribué à ce dernier : "La sûreté dépend d'une mutuelle correspondance à empêcher les pirates et courses des particuliers, qui, au lieu de s'appliquer en leur navigation à l'honnête exercice du commerce, rompent avec violence le lien de la société civile par lequel les nations se secourent les unes les autres en leurs nécessités. ", cité dans CLÉMENT, Pierre (éd.), Lettres, instructions et mémoires de Colbert, Paris, Imprimerie Impériale, 1854, t. I, p. 489.

24. Colbert s'appuie au début des années 1660 sur une estimation extravagante du nombre de gens de mer en France (MEYER, Jean, Colbert, op. cit., p. 261). Ce n'est que par le système des classes que Seignelay finit par admettre, en 1688, le nombre total de marins 
véritable capacité à fournir des matelots au moyen d'un dénombrement précis et catégorié : nombre de matelots recensés par havre, nombre de matelots embarqués à la pêche, en marchandise ou pour le service du roi, le niveau local de l'embauche maritime pour envoyer les matelots au chômage sur d'autres sites portuaires et leur ôter toute "oisiveté ". Ce recensement statistique des matelots annonce bien évidemment l'établissement du système des classes de la marine projeté par Colbert du Terron, instauré dès 1669, puis généralisé par une série de textes réglementaires et législatifs ${ }^{25}$. Bien entendu, les officiers des sièges d'amirauté de Normandie se gardent bien de procéder à pareil recensement des gens de mer, par la crainte d'une " remue " à l'instar de celle survenue à Touques ${ }^{26}$ :

"Aussy tost que nous avons esté honorés de votre ordre nous nous sommes transportés suivant icelluy au passage de Toucque qui est l'emboucheure de la mer parroisse de Trouville auquel lieu estant, bien loing de trouver des personnes soubmises aux ordres du Roy et de nous recepvoir comme gens venants de sa part et de la votre au grand mespris de l'arrest du conseil et de votre missive, nous ont voullu mollester et jeter dans la mer ce qu'ils eussent faict n'eust esté quelque secours qui nous a esté donné27."

\section{Des attentes fortement déçues}

L'instruction manuscrite de Colbert est bien peu suivie d'effet. Tous les procès-verbaux reçus des sièges d'amirauté normands et conservés dans le SH 48 cantonnent le détail de leurs rapports aux seuls mandements portés par l'arrêt du Conseil. Cela étant, les officiers des sièges ne peuvent que se fier aux compétences des " plus anciens des lieux \& experts " retranscrites dans les dépositions des comparants. Les procès-verbaux se limitent ainsi à la description topographique consciencieuse des ports et havres de leur juridiction ${ }^{28}$, sans s'émanciper du formalisme juridique de leurs procédures. Pouvait-il d'ailleurs en être autrement avec des délais de réponse présentés comme brefs? À l'égal des lieutenants des amirautés

français qui ne dépasse pas 50000 à 60000 hommes (la population de Rouen atteint à peu près 89000 âmes en 1640 !), après l'exil des matelots protestants.

25. ZYSBERG, André, « La soumission du rivage aux volontés de l'État royal ", dans ACERRA, Martine, Poussou, Jean-Pierre, Vergé-Franceschi, Michel, Zysberg, André (dir.), État, Marine et Société. Hommage à Jean Meyer, Paris, Presses universitaires de Paris-Sorbonne, 1995, p. $439-455$.

26. «Les débuts du règne de Louis XIV correspondent, on le sait, à une difficile période de mise au pas administrative. L'esprit de Fronde n'est pas éteint, les élites citadines renâclent, le royaume entier continue à frémir. " (NICOLAS, Jean, La rébellion française 1661-1789, Paris, Gallimard, 2008, p. 160.)

27. BnF, Mélanges Colbert (dés. Mél. Colb.), 128 bis, fol. 761-762, lettre du $1^{\mathrm{er}}$ avril 1665 des officiers de l'amirauté de Touques à Colbert.

28. "Nous avons pris le rapport et dressé un procez verbal de l'estat du havre de Barfleur le plus exact et le plus véritable quil nous a esté possible... " (BnF, Mél. Colb., 128 bis, $\mathrm{f}^{\circ} 738$, les officiers du siège de Barfleur à Colbert, lettre d'avril 1665.) 
de Rouen et de Cherbourg ${ }^{29}$, les autres officiers des amirautés normandes font généralement preuve de zèle administratif pour retourner leur procèsverbal le plus rapidement possible à Colbert ${ }^{30}$.

Durant les cinq semaines employées entre fin mars et début mai 1665 par les officiers des sièges pour effectuer leur visite et dresser le procèsverbal, les jours de visite d'une rade connue pour son marnage important sont occurrents avec de forts coefficients de marée (sièges de Coutances, Cherbourg, Bayeux, Barfleur, La Hougue, Saint-Valéry-en-Caux), de manière à pouvoir localiser précisément les éventuels dépôts anarchiques de lest. En revanche, les problèmes d'envasement ou d'invasion du galet étant déjà identifiés et localisés depuis longtemps par les comparants des grands ports de la généralité de Rouen, la tenue d'une visite un jour de coefficient de marée élevé ne paraît pas nécessaire (Dieppe, Honfleur, Rouen).

Aucun des renseignements fournis par les procès-verbaux n'est à négliger, à commencer par l'analyse des comparants désignés d'office par les lieutenants des sièges de Normandie. Leur nombre total s'élève à au moins 160 personnes, dont 38 à Dieppe. L'âge déclaré par les comparants constitue un élément de témoignage important car il véhicule la mémoire du littoral et de son éventuelle évolution naturelle. On obtient par exemple des témoignages sur l'effondrement des trafics durant le dernier conflit ou bien la confirmation du comblement progressif par les bancs de sable des petits havres du Coutançais depuis le Xvi ${ }^{\mathrm{e}}$ siècle $^{31}$. Ainsi, au havre de la « berque à l'eau " d'Agon, les comparants

" se souviennent que ledit havre a esté plus facille en son entrée qu'il n'est de présent et quil y en est peu entrer des navires de soixante et quatre vingt tonneaux et que l'emboucheure de la rivière qui fait séparation du havre de Regneville et dudit havre d'Agon a esté remplie de beaucoup de sables qui ont rendu l'ouverture moins creuse quoyque l'impétuosité de la mer et des vents jettent lesdits bancqs et destournent ladite rivière de profondeur dordinaire environ trois a quatre pieds, tantost d'un costé, tantost de l'autre ".

29. «Monseigneur, aussy tost vostre ordre receue avecq Larrest du Conseil Destat nous navons manqué a Lexecuter dont nous vous envoions le procez verbal que nous avons faict... " (BnF, Mél. Colb., 128 bis, fol. 797, Delafontaine à Colbert, lettre du 4 avril 1665). " Je nay manquay incontinent d'en dresser un le mieux qu'il a esté possible de l'estat auquel est le port de cette ville de rouen [...] le plus conforme a la volonté du Roy que jay peu. " (Ibid., f ${ }^{\circ} 824$, lettre de Deshoulley de Rouen à Colbert du 9 avril 1665.)

30. Les officiers font preuve de zèle dans les enquêtes de la flotte de 1664 (MORINEAU, Michel, "La Marine... ", op. cit., p. 240).

31. À Saint-Valéry-en-Caux, le lest que les vaisseaux " auroient par cy devant apporté n'a peu apporter aucune empirance audit havre pour n'avoir esté jetté dans le canal aiant mis en lieu qui na peu causer aucune incommodité ni dépérissement dudit havre estant currier en partie a raison du peu de vaisseaux qui y seroient entrés pendant la guerre..." (SHD Vincennes, SH 48). Sur la formation des havres du Cotentin occidental de Carteret à Granville (côte basse et cordon dunaire, rivière à débit suffisant, courant côtier ou dérive littorale), voir BouRnÉRIAS, Marcel, PoMEROL, Charles et TuRQuIER, Yves, La Manche du Havre à Avranches, guide n II, Neuchâtel, Delachaux \& Niestlé, 1984, p. 226-230. Sur la dérive littorale et sa fonction d'alimentation des plages en matériaux, voir PASKOFF, Roland, L'érosion des côtes, Paris, PUF, 1981, p. 16-17. 
Tableau 2 - Dates d'établissement des procès-verbaux des amirautés

\begin{tabular}{|c|c|c|}
\hline Ports & Date du procès-verbal & $\begin{array}{c}\text { Coefficient de marée (PV } \\
\text { occurrent) }\end{array}$ \\
\hline Dieppe & $\begin{array}{l}\text { 24/03/1665 (audition de Jacques de } \\
\text { Serne), } 26 \text { et } 27 / 03 / 1665 \text { (marchands et } \\
\text { capitaines réajournés) }\end{array}$ & $\begin{array}{c}55-50(24), 44-47 \text { (26) } 53-61 \\
\text { (27) }\end{array}$ \\
\hline Honfleur & $\begin{array}{c}\text { 25/03/1665 (lettre Colbert envoyée le } \\
08 / 03 \text { ) }\end{array}$ & $46-43$ \\
\hline Rouen & $\begin{array}{l}\text { 26/03/1665 (quais de Rouen) et } \\
\text { 27/03/1665 (de la porte Saint Éloy à la } \\
\text { Mailleraye) }\end{array}$ & - \\
\hline Bayeux & $\begin{array}{l}\text { 30/03/1665 (Port et Sainte-Honorine) et } \\
\text { 31/03/1665 (Arromanches et Annelles) }\end{array}$ & 102-107 (30), 111-113 (31) \\
\hline Carentan & $\begin{array}{l}\text { 31/03/1665 (Four de Taute et embou- } \\
\text { chure) et 08/04/1665 (Saint-Fromond) } \\
\text { (AC + lettre Colbert reçus le 28/03) }\end{array}$ & - \\
\hline Caudebec & $01 / 04 / 1665$ & - \\
\hline Caen & $\begin{array}{l}\text { 01/04/1665 (Caen) et 10/04/1665 (de } \\
\text { Colombelles au hameau de Longueval) }\end{array}$ & - \\
\hline Cherbourg & $\begin{array}{l}\text { 01/04/1665 (le havre) et 02/04/1665 (la } \\
\text { rade). Lettre de Colbert du 18/03 }\end{array}$ & $114-113(01), 110-106(02)$ \\
\hline Grandcamp & $\begin{array}{c}\text { 08/04/1665 (AC et lettre de Colbert du } \\
\text { 18/03/1665) et 09/04/1665 (rivière et } \\
\text { havre d'Isigny) }\end{array}$ & $34-31(08), 31-34(09)$ \\
\hline $\begin{array}{l}\text { Eu-Le } \\
\text { Tréport }\end{array}$ & $\begin{array}{c}\text { 11/04/1665 (lettre de Colbert envoyée le } \\
17 / 03)\end{array}$ & $49-55$ \\
\hline Barfleur & $15 / 04 / 1665$ & $87-90$ \\
\hline $\begin{array}{l}\text { La Hougue } \\
\text { \& Quinéville }\end{array}$ & $17 / 04 / 1665$ & 92 \\
\hline $\begin{array}{l}\text { Saint-Valéry- } \\
\text { en-Caux }\end{array}$ & $17 / 04 / 1665$ & 92 \\
\hline Coutances & $\begin{array}{l}\text { 24/04/1665 (havre de Regnéville). } \\
\text { 25/04/1665 (havres de Lingreville et } \\
\text { Bricqueville). 29/04/1665 (havre de } \\
\text { Coutainville et de la berque à l'eau à } \\
\text { Agon). 01/05/1665 (havres de Saint- } \\
\text { Germain et de Pirou) }\end{array}$ & $\begin{array}{c}48-51(24), 57-63(25), 103-105 \\
(29), 101-97(01)\end{array}$ \\
\hline $\begin{array}{l}\text { Ouistreham } \\
\text { \& Bernières }\end{array}$ & $\begin{array}{c}\text { 01/05/1665 (lettre Colbert envoyée le } \\
16 / 03 \text { ) }\end{array}$ & $103-101$ \\
\hline
\end{tabular}

Source : SHD Vincennes, SH 48. AC : arrêt du Conseil. Les coefficients de marée donnés (TU+2 heures) dans le tableau correspondent au jour de la visite des officiers d'amirauté et sont obtenus en ligne auprès du Service Hydrographique et Océanographique de la Marine de Brest. BnF, Mél. Colb., 128 bis, f ${ }^{\circ}$ 761, les officiers de l'amirauté de Touques prétendent dans leur lettre du $1^{\mathrm{er}}$ avril 1665 à Colbert avoir mis leur procès-verbal à la diligence pour Paris, mais on n'en a gardé aucune trace. L'absence de procès-verbal du siège de Dives s'explique par sa décadence administrative avancée d'après Joachim Darsel ${ }^{a}$. Au lieu d'un rapport de l'amirauté du Havre, on trouve dans le SH 48 un mémoire envoyé à Colbert par l'intendant Jean 
Baptiste Voysin de La Noiraye. Entré en fonction dans la généralité de Rouen depuis mai 1664, il inspecte souvent les travaux en cours dans les principaux ports de la généralité de Rouen (Dieppe, Le Havre, Honfleur) lors de l'enquête des amirautés de 1665. Son mémoire mentionne les aménagements à apporter au port du Havre et à la rade du Hoc illustré de deux cartes d'accompagnement du cartographe dieppois Guillaume Levasseur de Beauplan ${ }^{\mathrm{b}}$.

a. DARSEL, Joachim, «L'Amirauté en Normandie. L'amirauté de Dives ", Annales de Normandie, $26^{\mathrm{e}}$ année, $\mathrm{n}^{\circ} 2,1976, \mathrm{p} .114$.

b. BnF, Mél. Colb., 128 bis, f ${ }^{\circ}$ 885, lettre du 15 avril 1665 et Esmonin, Edmond, Mémoire..., op. cit., p. 9, n. 3. SHD Vincennes, Marine, SH 48, p. 65-67 pour les plans du Havre et du Hoc.

L'un d'eux affirme avoir appris de feu Jullien Le Marinel, son père, ancien matelot, qu'il entrait autrefois dans le havre de Lingreville des navires de 60 tonneaux. Malheureusement, les informations les plus précises sur l'âge des comparants se limitent aux seuls sièges de Caen (moyenne sur 13 individus : 62 ans, [âges extrêmes : 76 ans-40 ans]), de Carentan (moyenne sur 5 individus : 56, [80-30]) et de Coutances (moyenne sur 22 individus : 56, [94-40]). Les parcours professionnels des matelots et maîtres interrogés mentionnent un âge d'accès précoce aux métiers de la navigation et de la pêche, conforme aux us et coutumes des communautés littorales ${ }^{32}$. Ainsi, à Regnéville, Philippe Allain, âgé de 85 ans, navigue depuis 70 ans. Dans le havre de Linverville, Germain Noël, âgé de 64 ans, navigue aussi depuis l'âge de 12 ans. Jean Chevreüil, âgé de 94 ans, est maître et propriétaire de bateaux depuis l'âge de 20 ans. Grâce à cette accumulation d'informations diverses reçues des sièges d'amirauté, il est possible de dresser l'inventaire portuaire presque complet de la Normandie.

\section{L'inventaire des sites portuaires de Normandie}

\section{Préparer la guerre maritime en Manche}

Colbert songe dès son accession à la direction officieuse des affaires de la Marine en 1661 à faire rechercher des sites naturels ou portuaires capables d'abriter une flotte et son infrastructure navale mais la nouvelle rivalité anglo-hollandaise précipite sa décision. Le désaccord entre ces deux puissances maritimes porte à nouveau sur la pêche et la commercialisation du hareng ${ }^{33}$, " un si grand différend et si important pour ces États " (Isaac Bartet à Hugues de Lionne), mais s'étend à une violente compétition commerciale et coloniale (prises du fort de Cormantin, de l'île de Boa Vista et de la Nouvelle-Néderlande). Le déclenchement du conflit anglo-néerlandais en mars 1665 préoccupe à juste titre le Conseil royal tant par les pré-

32. Par exemple, les enfants des pêcheurs granvillais commencent à travailler à partir de 8-9 ans (CABANTOUS, Alain, Les citoyens du large. Les identités maritimes en France (XVII XIX $X^{e}$ siècle), Paris, Aubier, 1995, p. 59).

33. Unger, Richard W., " Dutch Herring, Technology, and International Trade in the Seventeenth Century ", The Journal of Economic History, vol. XL, n ${ }^{\circ}$, 1980, p. 253-279. 
paratifs d'armement d'une centaine de vaisseaux de la Royal Navy, la presse des équipages signalée de décembre 1664 à mars 1665 à Chatham ${ }^{34}$, que par les ambiguïtés sous-jacentes des pays protagonistes. Pourtant alliées à Louis XIV, les Provinces-Unies commencent à s'inquiéter ouvertement des ambitions commerciales françaises ${ }^{35}$.

La "furieuse jalousie " anglaise envers la France préoccupe tout autant Louis XIV. L'Angleterre s'est lancée depuis 1636 dans le fructueux trafic avec les Indes orientales, construit méticuleusement sa flotte de guerre, bien après avoir revendiqué la domination de ses mers bordières jusqu'aux rivages opposés (Manche, mer du Nord) ${ }^{36}$. De leur côté, les marchands de la City craignent ouvertement depuis 1662 la rapide montée en puissance du commerce français, attestée par une balance des échanges de plus en plus défavorable à l'Angleterre ${ }^{37}$. Enfin, les compagnies privilégiées, anglaise comme hollandaise (Society of merchants venturers, WIC), en pleine rivalité pour dominer le grand commerce atlantique ${ }^{38}$, ne veulent surtout pas souffrir la présence du nouveau concurrent français dans ce secteur.

Colbert a donc ordonné dès 1662 la recherche de sites idoines afin d'établir des ports de guerre capables de rivaliser avec Southampton et surtout, Portsmouth ${ }^{39}$. Il penche en 1663 en faveur du Havre pour " la construction d'un outil maritime " en Normandie, sans négliger les autres côtes du royaume $^{40}$. Les travaux de Rochefort, "l'enfant chéri de Colbert ${ }^{41}$ ", débutent en 1663. Le choix plus réaliste de Brest se concrétise l'année suivante lors de la chevauchée de Colbert du Terron. L'ogre dunkerquois se pré-

34. Rogers, Philip C., The Dutch in the Medway, Londres, Oxford univ. press, 1970, p. 41-42. Sur ce conflit maritime, "terrible, obstinate and bloody battle ", voir RoDGER, Nicholas Andrew Martin, The Command of the Ocean. A naval history of Britain, 1649-1815, Londres, Penguin books, 2004, p. 65-79.

35. Bots, Hans, "L'Image de la France dans les Provinces-Unies ", dans Méchoulan, Henry et CORnETTE, Joël (dir.), L'État..., op. cit., p. 346. JonEs, James R., The Anglo-Dutch wars of the Seventeenth century, Londres-New York, Longman, 1996, p. 182.

36. HARDING, Richard, Seapower and Naval Warfare 1650-1830, Londres, UCL press, 1999, p. 59-120. Poussou, Jean-Pierre, Les Îles britanniques, les Provinces-Unies, la guerre et la paix au XVII siècle, Paris, Economica, 1991, p. 77-82.

37. Sur la deuxième guerre maritime anglo-hollandaise et le rôle naval ambigu de Louis XIV dans la réaction hollandaise en 1666 : HASQUIN, Hervé, Louis XIV face à l'Europe du Nord, Bruxelles, éd. Racine, 2005, p. 118-121. LEVILLAIN, Charles-Édouard, Vaincre Louis XIV. Angleterre-Hollande-France : histoire d'une relation triangulaire 1665-1688, Seyssel, Champ Vallon, 2010, p. 90-93 et 155-156. DAvis, Ralph, " English Foreign Trade, 1660-1700 ", dans Minchinton, Walter E. (ed.), The Growth of English Overseas Trade in the Seventeenth and Eighteenth Centuries, Londres, Methuen \& Co., 1969, p. 78-98.

38. Jones, James R., The Anglo-Dutch wars..., op. cit., p. 147.

39. Colbert du Terron, intendant de la Marine du Ponant parcourt à la fin de 1664 les rivages de la Manche à la recherche d'un site favorable (VERGÉ-FrANCESCHI, Michel, Abraham Duquesne..., op. cit., p. 205). Son rapport final est conservé aux Archives de la Marine, $\mathrm{D} / 27$.

40. Peter, Jean, L'arsenal du Havre..., op. cit., p. 14.

41. Sur le choix de la construction de Rochefort : Meyer, Jean et Poussou, Jean-Pierre, Études sur les villes françaises. Milieu du XVII siècle à la veille de la Révolution française, Paris, éd. SEDES-CDU, 1995, 2 éd., p. 256. 
pare à engloutir au total douze millions de livres en fortifications, chenaux, digues, bassins, écluses ${ }^{42}$. Le littoral normand, idéalement orienté face aux côtes anglaises, peut-il accueillir un port de guerre? Rien n'est moins sûr. Malgré la clairvoyance de l'ingénieur Baudouyn, Colbert ne manifeste aucun intérêt pour la rade de Cherbourg. Dieppe ne compte finalement pour rien malgré la "bonté " de sa rade.

Après d'épiques foires d'empoigne entre ingénieurs, échevins, commissaires, officiers de marine et l'encombrant Duquesne ${ }^{43}$, l'utopie navale havraise, aussi médiocre que fort coûteuse, passe au bout du compte par pertes et profits. D'après l'intendant Voysin de La Noiraye, l'obstacle de loin le plus contraignant est que tous les ports normands sont " havres de marée et ou mesme l'on n'entre que de vive-eau ", à l'exception de la fosse d'Omonville (Manche) ${ }^{44}$. D'après l'ingénieur architecte Gobert, l'inconvénient de tous les ports de Normandie orientale, depuis Le Havre jusqu'au Tréport, et même jusqu'à Dunkerque, est que « les jetées ne sont pas suffisamment avancées. À la mer montante, le flot provoque une accumulation de galets ou pouliers que les barres ne peuvent chasser ${ }^{45}$ ".

Le SH 48 permet alors de recenser les facteurs naturels qui expliquent le désintérêt final de la monarchie envers le littoral normand. On ne peut, sur le fond, mettre en doute la bonne foi des officiers des sièges d'amirauté, pourtant largement insérés dans les réseaux de réciprocités sociales et de solidarités locales ${ }^{46}$. En revanche, les comparants ont vite compris tout l'avantage à retirer d'éventuelles retombées économiques locales en cas de construction d'un nouveau port. Ceux de Saint-Valéry-en-Caux affirment par exemple " que la rade du havre de Saint-Vallery est l'une des meilleures rades des ports de France, et la plus asseurée de la coste de Normandie ", une affirmation d'ailleurs partagée par Duquesne, mais pour la rade de Fécamp ${ }^{47}$. En réalité, il ne s'agit que d'un simple mouillage à l'ouest de

42. Le Havre absorbe de 1667 à 1705 entre trois et cinq millions de livres en infrastructures et fortifications du port et de l'arsenal, crédits surtout consommés entre 1665 et 1672 (PETER, Jean, L'arsenal du Havre..., op. cit., p. 216; MEYER, Jean, Béveziers (1690). La France prend la maîtrise de la Manche, Paris, Economica, 1993, p. 26).

43. Le conflit devient ouvert entre Colbert du Terron, Regnier Jansse, le duc de Saint-Aignan et les élites marchandes havraises d'une part, et Duquesne, devenu le conseiller le plus écouté de Colbert dans l'aménagement des ports en Normandie d'autre part (PETER, Jean, L'arsenal du Havre..., op. cit., p. 16 et 38; VerGé-FrAnCESCH, Abraham Duquesne ..., op. cit.., p. 206-216).

44. Le rapport de l'ingénieur hollandais Regnier Jansse le jeune, qui accompagne Colbert du Terron en 1664, mentionne sa profondeur de 12-16 pieds d'eau au moins, son accessibilité à toute heure de marée et sa capacité à abriter 30 vaisseaux de guerre.

45. Peter, Jean, L'arsenal du Havre..., op. cit., p. 18. Sur les mécanismes de l'érosion des falaises en Haute-Normandie : HÉNAFF, Alain, LAGEAT, Yannick, CosTA, Stéphane et Plessis, Emmanuelle, "Le recul des falaises crayeuses du Pays de Caux : détermination des processus d'érosion et quantification des rythmes d'évolution/Retreat of chalk cliffs in the Pays de Caux : processes and rates ", Géomorphologie : relief, processus, environnement, avril-juin 2002, vol. 8, ${ }^{\circ}$ 2, p. 107-118.

46. JACQUART, Jean, "Colbert... ", op. cit., p. 189 et MEYER, Jean, Colbert, op. cit., p. 54 et 59.

47. « La rade de ce lieu est suffisamment profonde et le fond y est bon pour les ancres; elle est sans abry depuis le nord jusqu'au surouest, comme toutes les autres rades depuis 
l'entrée de Saint-Valéry. Il est donc permis de douter, même partiellement, de l'objectivité des dépositions.

\section{L'orientation des sites portuaires}

La méthode critique la plus pertinente consiste à retenir les trois principaux critères d'analyse des sites portuaires présentés par Edmond Esmonin (orientation, profondeur et qualité du fond des rades) tout en croisant dès que possible les informations du SH 48 avec d'autres sources. L'orientation de l'entrée du port dans le secteur favorable du sud-sud-ouest, facilitant l'entrée d'un vaisseau au port ou pour sortir de la Manche, constitue le premier critère à une époque où les navires au long cours ne disposent que de la seule énergie éolienne. Ce point crucial est pourtant peu évoqué dans les rapports, à l'exception des procès-verbaux de naufrages qui évoquent dans le cas du Havre, par exemple, "la puissance de la lamme " contre les jetées lors des tempêtes (voir figure en hors-texte) ${ }^{48}$. Des contemporains notent d'ailleurs de plus en plus scrupuleusement les épisodes et phénomènes météorologiques dévastateurs qui frappent avec plus ou moins de régularité le littoral, en raison de leurs répercussions financières sur le commerce maritime, et plus généralement sur les biens publics et privés ${ }^{49}$.

C'est donc de bonne guerre lorsque chaque marchand cherche à attirer à lui une clientèle de correspondants portuaires, en vantant par exemple la supériorité nautique des accès d'Honfleur sur ceux du Havre :

"Il y a moins de risques à venir à Honfleur qu'à entrer au Havre, de foudre de vent. Les navires n'entrent point au Havre sans risquer beaucoup d'être perdus en entrant contre les jetées, et même dans le Havre, à cause que la mer y est toujours fort agitée (les navires s'entrecrèvent) des vents de sudouest et ouest-nord-ouest, qui est le vent propre pour arriver ${ }^{50}$."

Mais n'en déplaise à notre commissionnaire en morues d'Honfleur, Le Havre et Le Tréport offrent seuls cette avantageuse orientation des

\footnotetext{
le Havre jusques à Calais et Dunkerque. " (BRÉARD, Charles, " Mémoires... ", op. cit., p. 18.)

48. Langlois de Collemoulins signale en 1627 que " la passe est difficile " pour l'entrée et la sortie du port du Havre (Coluins, James B., " La flotte normande au commencement du XVII e siècle : le mémoire de Nicolas Langlois (1627) ", Annales de Normandie, $34^{e}$ année, $\mathrm{n}^{\circ} 4$, 1984, p. 366). SHD Vincennes, Marine, fonds Nivart, $\mathrm{ms} \mathrm{n}^{\circ} 144$, p. 302, plan explicatif de la submersion de la frégate la Galathée dans la passe du Havre lors de la tempête du 26 septembre 1708 à 7 heures du soir par une tempête du sud-ouest.

49. DESARTHE, Jérémy, « Les sociétés face aux caprices du temps dans l'Ouest de la France ( $\mathrm{xvI}^{\mathrm{e}}$-XIX ${ }^{\mathrm{e}}$ siècle) ", dans Ducos, Joëlle, Le temps des saisons. Climat, événements extrêmes et sociétés dans l'Ouest de la France (XVI-XIXe siècles), Paris, Hermann, 2013. Autre manifestation d'accidents climatiques : des tempêtes cycloniques hivernales ravagent les accès du port du Havre en novembre 1662, ceux d'Honfleur en décembre 1665 (Esmonin, Edmond, Mémoire..., op. cit., p. 192-194). ReICH, Christina, Der "Great Storm" 1703. Zur Geschichte einer Naturkatastrophe zu Beginn des 18. Jahrhunderts in England, Bonn, Minifanal, 2013.

50. Le marchand-armateur terre-neuvien Charles Lion d'Honfleur à l'armateur malouin Granville-Locquet, 27 juin 1691 (DEChARME, Paul, Le comptoir d'un marchand au XVII siècle, Paris, Hachette \& Cie 1910 , p. 123).
} 

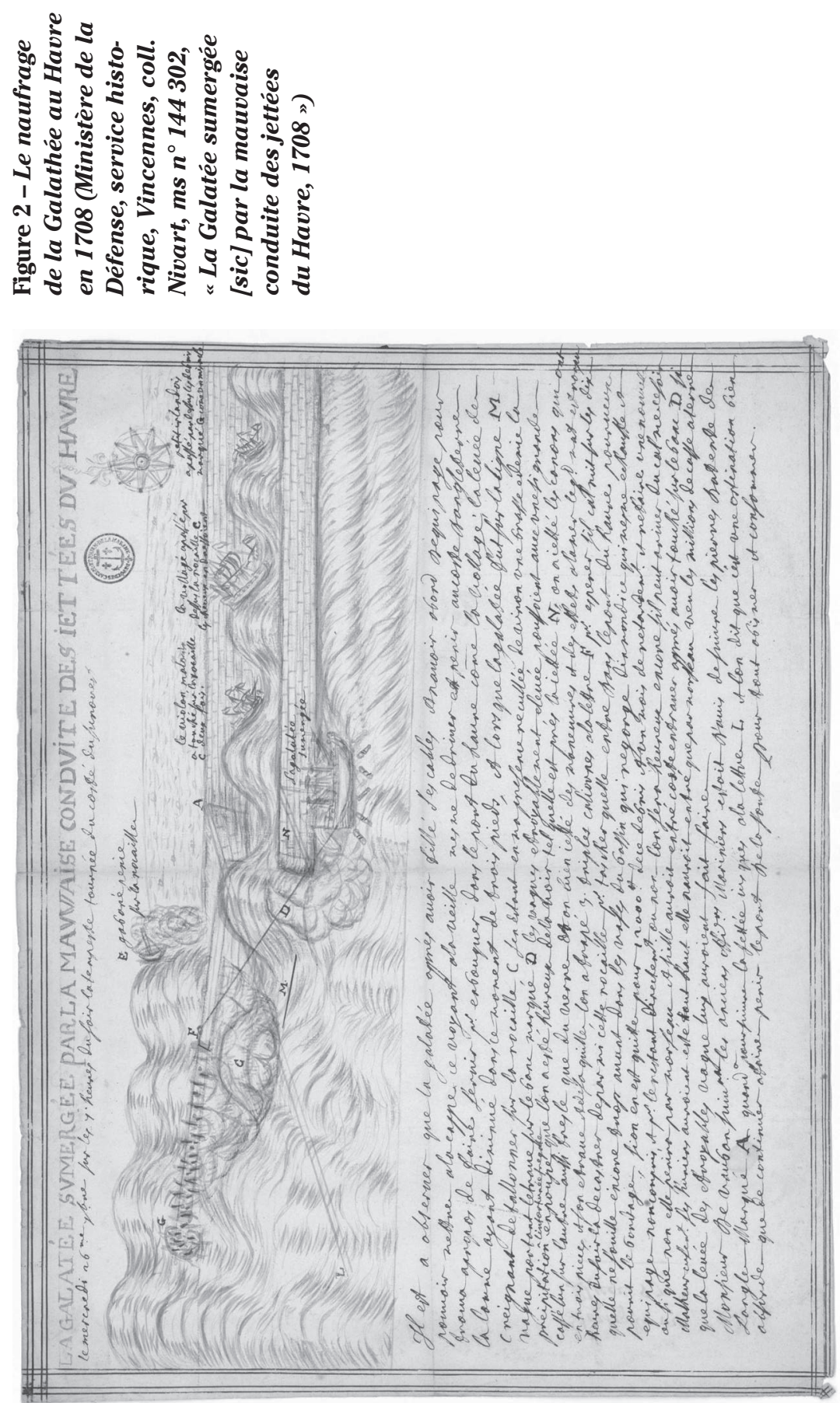
vents. La grande rade du Tréport est orientée ouest/sud-ouest à une grande lieue du port. La petite rade se située "à portée de mousquet " (120 à 150 toises, soit 300 m environ). Le problème est qu'en cas de tempête, les navires dépourvus de protection doivent impérativement dérader après avoir coupé câbles, ancres, amarres et faire route vers d'autres rades au sud-ouest ou au nord du Tréport. L'entrée du Havre est bien orientée pour recevoir les vents portants mais son exposition l'expose à l'engorgement régulier de ses accès par le galet. Dès 1662, le chevalier de Clerville ne le considère pas comme un port " propre pour les navires du roi, ou de grand port. ". Les travaux qu'il estime nécessaires ne peuvent que le rendre bon aux navires marchands jusqu'à 300-600 tx. Duquesne juge également les rades du Havre très médiocres et dangereuses lors de son inspection de 1665 :

"Aujourdhuy au meilleur endroit où il y a très peu d'espace il ne se trouve de basse marée au plus qu'environ vingt pieds de profondeur, et il est arrivé souvent qu'un navire de médiocre grandeur, lors d'un vent de tempeste ou les vagues de la mer sont grandes que les dictz vaisseaux ont touché entre le fond, ce qui les a contraint de s'abandonner à la coste, en sorte qu'il ne faut faire estat de la dicte rade que pour peu de vaisseaux de deux cens tonneaux ${ }^{51}$."

De son côté, l'ingénieur François Blondel affirme, dans son devis du 10 décembre 1664, que « les incommodités de la rade et de l'entrée du Havre sont telles qu'il n'y a pas d'apparence que l'on veuille s'en servir pour les grands vaisseaux du roi ". Il préconise un simple " raccommodage " des écluses, le nettoyage du bassin et la réfection des épis de l'estran pour bloquer la progression du galet vers l'entrée du port ${ }^{52}$. La rade du Hoc (voir figure $\mathrm{n}^{\circ} 3$ ) située à l'embouchure de la rivière d'Harfleur a un fond de bonne tenue pour l'ancrage et de profondeur suffisante (25 à 26 pieds) mais sa sortie est mal orientée vers le sud-est. La mer se montre de plus " extraordinairement émue " en cas de vent d'ouest contre le courant de jusant. Les vaisseaux au mouillage sur la rade y fatiguent alors considérablement. De plus, les navires ne peuvent séjourner en grand nombre dans ce mouillage trop exposé aux vents de secteur ouest et nord-ouest ${ }^{53}$.

En Basse-Normandie, la rade de Cherbourg dite du Gallet est le lieu où " possent » les navires, c'est-à-dire jettent l'ancre à l'échouage en l'attente de pouvoir entrer dans le port. Cette vaste rade est considérée par les comparants comme assez bonne de secteur de vent de sud à sud-ouest, un avis sérieusement contredit à trente ans de distance par le subdélégué de l'élection de Valognes ${ }^{54}$. En effet, son orientation ne permet pas aux

51. BRÉARD, Charles, "Mémoires... ", op. cit., p. 11.

52. PETER, Jean, L'arsenal du Havre... op. cit., p. 2 et 9-10.

53. BnF, ms. fr. 18596. Langlois de Collemoulins souligne en 1627 qu'en rade du Havre les " vents d'oest (ouest), sorest (suroît), norest (noroît) sont des vents qui chassent en coste et qui causent des nauffrages".

54. En 1627, les pratiques du lieu trouvent le " port et havre assez bon et est a labry des vents " (Ibid.). " La rade n'est pas trop bonne, l'ancrage n'y est pas seur, et le port trop 

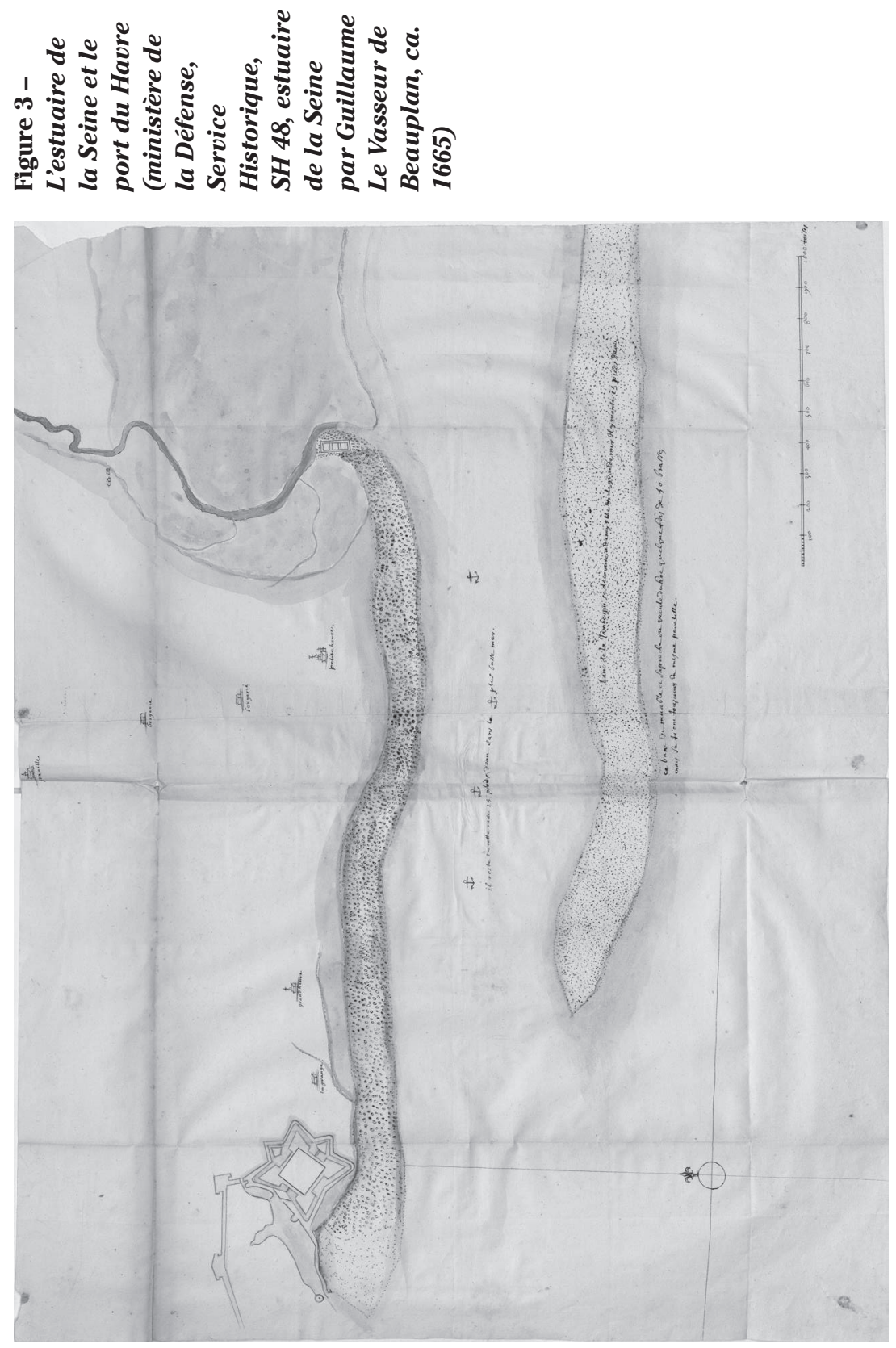

exposé aux vents d'ouest et sud-oüest [...] La rade n'est pas trot bonne, le vent d'ouest et suroit luy sont contraire, le tout ni est pas ben \& il ni a pas bonne teneure. " (GouHIER, Pierre, L'Intendance de Caen en 1700, Paris, éd. CTHS, p. 238 et 343). En 1688, Seignelay constate que son havre se remplit continuellement de sables (Ibid., p. 131, n. 137). 
navires d'en sortir sous ce secteur, car obstrué par la terre et les roches du Hommet dressées à proximité et qui abritent la rade de ces vents. Lorsque le vent est établi à l'est/nord-est les vaisseaux peuvent rader en se mettant à " l'abruit " de l'île Pelée, éloignée d'environ une demi-lieue du havre du Gallet et où l'on retrouve les profondeurs de 72 pieds et 36 pieds.

Dans le Coutançais, les rades et havres de Linverville, Gonneville et Gouville ne peuvent servir d'abri en raison de leur orientation ouverte face aux tempêtes d'ouest, en dépit de leur profondeur suffisante : le marinier Germain Noël de Linverville a " sondé la rade en morte eau et marée commune avec les cordes et le plomb et que dans les raddes où d'ordinaire vont les batteaux de cette coste il y a viron quarante pieds de haulteur et dans les marées communes et au plain d'icelle viron vingt quatre pieds, que toutes sortes de navires y pouroit radder mesme durant le plain de la morte eau néantmoins que ce ne soit un lieu de seuretté devant les tempestes des ventz de ouetz sur ouestz et du sut nous attestant qu'il n'a jamais veu de changement dans lesdittes raddes. " En conclusion, très peu de ports normands sont susceptibles d'offrir une orientation convenable. Il en va de même avec l'amplitude des marées.

\section{Un obstacle à l'accessibilité portuaire des flottes de guerre : les amplitudes de marées}

Le marnage d'un site portuaire, ou amplitude maximale entre la haute et la basse mer, doit être le plus réduit possible selon Voysin de La Noiraye, de manière à permettre l'entrée ou la sortie la plus fréquente possible des vaisseaux. Or, la configuration des côtes de la Manche accroît le phénomène de marnage dans des proportions très importantes au fur et à mesure que l'on progresse d'est en ouest. Ainsi, le maximum absolu du marnage est atteint au Mont-Saint-Michel, décroît ensuite au niveau du méridien de Cherbourg, avant de croître à nouveau vers l'est, mais de manière bien moins prononcée $^{55}$. Les petits ports, havres et rades du Cotentin, du Coutançais et de l'Avranchin sont de facto exclus des sites à retenir pour le projet de Colbert. En revanche, l'avantageuse situation géographique de La Hougue, Porten-Bessin et Ouistreham les surclasse par rapport aux ports cauchois ou picards dans le système des marées en Manche ${ }^{56}$.

55. Le fort marnage découvre à basse mer d'immenses franges du littoral du Cotentin occidental et engendre des courants marins très violents, notamment au raz Blanchard (jusqu'à 10 nœuds, soit 18,52 km/h) en période de grande marée d'équinoxe (BouRNÉRIAS, Marcel et al., La Manche du Havre..., op. cit., guide II, p. 37-39).

56. " Les ports de basse Normandie ont un grand avantage sur tous ceux qu'on peut faire au pays de Caux et en Picardie, [...] les vaisseaux peuvent sortir de nos ports et entrer dans la Seine d'une même marée, au lieu qu'il faut que les vaisseaux qui sortent de la Picardie et du pays de Caux sortent d'une marée de leurs ports et en attendent une autre pour entrer dans la Seine, ce qui est toujours accompagné de quelque péril de la tempeste et ce qui les expose aux pirates ennemis " (GouHIER, Pierre, L'Intendance..., op. cit., p. 133). 
Les caractéristiques des marées relevées en Normandie (système alternatif semi-diurne, marées de vive-eau et de morte-eau, amplitude saisonnière des marées au cours de l'année et surtout aux équinoxes, ondes de tempête) influent directement sur les profondeurs des rades et ports de la province et, par voie de conséquence, sur les tirants d'eau des navires. La profondeur de référence minimale pour l'entrée des bâtiments de commerce dans les ports marchands est de 18,5 pieds (6,10 m), équivalente au port des vaisseaux de guerre de 500-600 tonneaux tout au plus (vaisseaux de $4^{\mathrm{e}}$ rang ${ }^{57}$. Clerville à Honfleur et Duquesne au Havre retiennent eux aussi ce seuil de 18 pieds, au-delà duquel il ne se trouve plus de port éligible dans le projet de Colbert ${ }^{58}$. Les profondeurs des ports, havres et rades indiquées dans les procès-verbaux du SH 48 entretiennent néanmoins à dessein l'illusion de leur accessibilité nautique aux pleines mers de vive-eau. Dans le port de Honfleur par exemple, on sonde 22 pieds de profondeur " ce qui donne lieu aux navires par la conduitte des pilottes lamaneurs dy entrer tous chargez et d'en sortir en mesme estat. " Clerville tempère pourtant l'enthousiasme du lieutenant du siège Amelyne, en précisant dans son rapport de 1664 à Colbert que « comme l'entrée n'en est pas trop bonne à cause des batures qui sont au devant, il n'y a gueres de lieu d'espérer qu'on y puisse introduire de grands navires ${ }^{59}$ ".

Le tableau 4 synthétise les dépositions des comparants sur les hauteurs d'eau de leurs ports, parfois corrigées ou complétées par le recours à d'autres sources. Les cases figurant en grisé représentent le seuil de référence de 18 pieds minimum atteint soit à la pleine mer de vive-eau, soit à pleine mer de morte-eau ou encore à basse mer de vive-eau (aucun procèsverbal n'indique la profondeur à basse mer de morte-eau, la plus faible).

Les cases en grisé du tableau font ressortir le nombre restreint de ports normands susceptibles d'atteindre, voire de dépasser, le seuil minimal de 18 pieds. Le Tréport, Dieppe, probablement le mouillage de Saint-Valéryen-Caux en pleine mer de vive eau, et Fécamp lorsque son port est débouché, sont les seuls ports du littoral cauchois capables de présenter une profondeur quelque peu acceptable ${ }^{60}$. Le chenal du Havre, régulièrement

57. À Dieppe, au XVII ${ }^{\mathrm{e}}$ siècle, les navires de 400 tx disposent d'un tirant d'eau de 14 pieds environ (GuIBERt, Michel-Claude, Mémoires pour servir à l'histoire de Dieppe, Dieppe, Paul Leprêtre imp., 1878, 2 vol., t. I, p. 249).

58. PETER, Jean, L'arsenal..., op. cit., p. 9 et 16. Clerville estime à 18-20 pieds au moins la profondeur nécessaire au projet de creusement d'un nouveau bassin à Honfleur (ESMONIN, Edmond, Mémoire..., op. cit., p. 197).

59. EsMONIn, Edmond, Mémoire..., op. cit., p. 197.

60. Fécamp pourrait accueillir des vaisseaux de 300 tx. au lieu de 100 tx., mais les installations du port (écluses, barres) relevant de l'abbaye bénédictine de Fécamp sont laissées à l'abandon (Esmonin, Edmond, Mémoire..., op. cit., p. 8 et n. 5). Nicolas Langlois de Colmoulins se rend de Dieppe à Saint-Valéry en passant par Veules " auquel n'y a que petitz basteaux pescheurs " (BnF, ms fr. 18596, f ${ }^{\circ}$ 7, le 25 avril 1627). À Saint-Valéry, Langlois procède à la " visitation " du havre qu'il trouve " tres bon et en bon estat par le moien des Ouvrages et jettéés y ont esté faicts depins xii a xiii ans en ça et pour son assiette a cause de la Coste et abbry des grandz vents daval quil y entre, vingt a vingt 
Tableau 4 - Hauteurs d'eau dans les ports de Normandie en 1665

\begin{tabular}{|c|c|c|c|c|c|}
\hline Ports & Site & $\begin{array}{l}\text { Hauteur } \\
\text { d'eau de } \\
\text { "grande } \\
\text { mer " en } \\
1627\end{array}$ & $\begin{array}{l}\text { Hauteur d'eau à } \\
\text { PM de vive-eau }\end{array}$ & $\begin{array}{l}\text { Hauteur } \\
\text { d'eau à PM } \\
\text { de morte- } \\
\text { eau }\end{array}$ & $\begin{array}{c}\text { Hauteur } \\
\text { d'eau, BM de } \\
\text { vive-eau }\end{array}$ \\
\hline Eu-Le Tréport & La Bresle & - & 20 pieds au moins & 12 pieds & - \\
\hline Eu-Le Tréport & Canal d'Artois (Tréport-Eu) & - & 15-16 pieds & - & - \\
\hline Eu-Le Tréport & Grande rade & - & 10 brasses & $\begin{array}{c}6 \text { brasses. } \\
\text { 5-6 brasses } \\
\text { (Voysin } \\
\text { de La } \\
\text { Noiraye) }\end{array}$ & \\
\hline Eu-Le Tréport & Petite rade & $\begin{array}{l}20 \text { pieds } \\
\text { à l'entrée } \\
\text { du port } \\
\text { (visite de } \\
1639 \text { ) }\end{array}$ & $\begin{array}{c}5 \text { brasses ( } 4 \text { selon } \\
\text { Voysin) }\end{array}$ & - & - \\
\hline Dieppe & La rade & $\begin{array}{l}20-24 \\
\text { pieds }\end{array}$ & $\begin{array}{l}20 \text { pieds (Gobert } \\
\text { en } 1665)\end{array}$ & - & $\begin{array}{c}\text { 7-8 brasses. } \\
\text { 8-9 brasses } \\
\text { (caps. } \\
\text { Barbier, } \\
\text { Soyer, Fillye). } \\
\text { 9-10 brasses } \\
\text { (lt. amirauté) }\end{array}$ \\
\hline $\begin{array}{l}\text { Saint-Valéry- } \\
\text { en-Caux }\end{array}$ & $\begin{array}{l}\text { Le havre de Saint-Valéry dit } \\
\text { les marestz }\end{array}$ & $\begin{array}{l}20-22 \\
\text { pieds }\end{array}$ & $\begin{array}{c}\text { Entrée en partie } \\
\text { bouchée par tem- } \\
\text { pête de } 1662.4 \\
\text { brasses (Voysin) }\end{array}$ & $12-13$ pieds & - \\
\hline Fécamp & $\begin{array}{c}\text { Entrée du port } \\
\text { "De cette manière le port, } \\
\text { qui a desjà de profondeur } \\
\text { à son entrée jusques à } \\
\text { vingt cinq pieds d'eau aux } \\
\text { grandes marées, reprendra } \\
\text { son ancienne largeur et se } \\
\text { nettoyera par le dedans, } \\
\text { que l'on y pourra tenir } \\
\text { comme autrefois plusieurs } \\
\text { vaisseaux flottant de basse } \\
\text { marée. " (Bréard, Charles, } \\
\text { "Mémoires... ", op. cit., } \\
\text { p. 18). }\end{array}$ & $\begin{array}{l}18-20 \\
\text { pieds }\end{array}$ & $\begin{array}{l}4,5 \text { brasses } \\
\text { (Voysin) }\end{array}$ & - & - \\
\hline Le Havre & Entrée du port & $\begin{array}{l}18-20 \\
\text { pieds }\end{array}$ & $\begin{array}{l}\text { 15-16 pieds d'eau } \\
\text { (1661 et 1668, R. } \\
\text { Jansse). 25-26 } \\
\text { pieds (Montegui) }\end{array}$ & $\begin{array}{c}10-11 \\
\text { pieds. } \\
\text { Bouchée } \\
\text { en } \\
\text { novembre } \\
1664 \\
\end{array}$ & - \\
\hline Le Havre & La rade & $\begin{array}{l}\text { Le Hoc : } \\
18-20 \\
\text { pieds }\end{array}$ & & - & $\begin{array}{c}3 \text { brasses } \\
\text { (Voysin) }\end{array}$ \\
\hline
\end{tabular}

deux piedz d'eau de grande mer Cy peut entrer des vaisseaux de trois a quatre cens tonneaux, Comme aussy fort ordinairement de ressauts aux Navires agittez de la Tampeste de la mer...". 
Les ports, havres et rivières navigables de Normandie en 1665

\begin{tabular}{|c|c|c|c|c|c|}
\hline Honfleur & La rade & - & - & - & $\begin{array}{c}3 \text { brasses } \\
\text { d'eau ou } 15 \\
\text { pieds tout au } \\
\text { moins }\end{array}$ \\
\hline Honfleur & Le canal ou ruel et port & $\begin{array}{l}18-20 \\
\text { pieds }\end{array}$ & $\begin{array}{c}20-22 \text { pieds/14-15 } \\
\text { pieds (Gobert, } \\
1665)\end{array}$ & - & - \\
\hline Port-en-Bessin & Le port & - & 18 pieds & - & - \\
\hline Port-en-Bessin & Grande et petite rades & - & & $\begin{array}{c}40 \\
\text { brasses/8-9 } \\
\text { brasses } \\
\text { (Gouhier, } \\
1746 \text { ) }\end{array}$ & \\
\hline $\begin{array}{l}\text { Sainte- } \\
\text { Honorine }\end{array}$ & - & - & 10 pieds & 8 pieds & - \\
\hline $\begin{array}{l}\text { Arromanches } \\
\text { \& Annelles }\end{array}$ & - & - & 6 pieds & - & - \\
\hline Grandcamp & Rade et banc du Sablon & - & 10-12 pieds & - & - \\
\hline Grandcamp & Ancien havre & - & $10-12$ pieds & - & - \\
\hline Grandcamp & Grouin de la dune & - & 30 pieds environ & - & - \\
\hline Grandcamp & Rivière et havre d'Isigny & - & 20 pieds & - & 4 pieds \\
\hline $\begin{array}{l}\text { Rivière de } \\
\text { Vire }\end{array}$ & - & - & $20-30$ pieds & & \\
\hline $\begin{array}{l}\text { La Hougue \& } \\
\text { Quinéville }\end{array}$ & - & 15 pieds & 14 pieds & 7 pieds & - \\
\hline Barfleur & Havre de Barfleur & - & $\begin{array}{c}13-14 \text { pieds } \\
\text { (embouchure et } \\
\text { milieu) }\end{array}$ & $10-11$ pieds & - \\
\hline Cherbourg & Le havre & 25 pieds & 18 pieds & 7 pieds & - \\
\hline Cherbourg & Rade du Gallet & - & 72 pieds & 36 pieds & - \\
\hline Port Bail & & 10 pieds & & & \\
\hline $\begin{array}{l}\text { Havre de } \\
\text { Regnéville }\end{array}$ & - & - & $10-12$ pieds & 4-5 pieds & - \\
\hline $\begin{array}{c}\text { Havre de } \\
\text { Lingreville et } \\
\text { Bricqueville }\end{array}$ & - & - & 10 pieds & - & - \\
\hline $\begin{array}{c}\text { Linverville, } \\
\text { Gonneville et } \\
\text { Gouville }\end{array}$ & - & - & 40 pieds & 24 pieds & - \\
\hline $\begin{array}{c}\text { Havre de } \\
\text { Coutainville }\end{array}$ & - & - & - & 8 pieds & - \\
\hline $\begin{array}{c}\text { Havre de la } \\
\text { barque à l'eau } \\
\text { (Agon) }\end{array}$ & - & - & - & 8 pieds & - \\
\hline $\begin{array}{c}\text { Havre de } \\
\text { Saint-Germain }\end{array}$ & - & - & 15 pieds & 6 pieds & - \\
\hline $\begin{array}{l}\text { Havre de } \\
\text { Pirou }\end{array}$ & - & - & $12-15$ pieds & - & - \\
\hline
\end{tabular}

Sources : SHD Vincennes, SH 48; PETER, Jean, L'arsenal..., op. cit.; Esmonin, Edmond, Mémoire..., op. cit.; BnF, ms fr. 18596, mémoire de Nicolas Langlois de Collemoulins, 1627 (Dieppe à Genêt, pas de renseignements sur Eu-Le Tréport, du ressort du Parlement de Paris). Métrologie : un pied vaut $0,33 \mathrm{~m}$. Une brasse marine ou " brasse nouvelle " vaut cinq pieds (1,624 m). Collins, James B., "La flotte normande... ", op. cit.; peu d'écart des profondeurs des ports à constater entre 1627 et 1665. 
" engorgé " par les vases et galets, atteint péniblement 15 à 16 pieds de profondeur à la pleine mer de vive eau et 10-11 pieds en morte eau. Honfleur affiche une profondeur plus convenable mais son port, partiellement envasé depuis des décennies, restreint le trafic avec une profondeur maximum de 14-15 pieds ${ }^{61}$. En Basse-Normandie, les ports disposant d'une rade profonde sont peu nombreux. Les ports et havres suivants ne sont du reste accessibles aux bâtiments de commerce qu'aux pleines mers de vive eau à chaque quinzaine : Port-en-Bessin, le mouillage forain du Grouin de la dune et le havre d'Isigny dans le ressort de l'amirauté de Grandcamp, la rade du Gallet à Cherbourg, les rades de Linverville, Gonneville et Gouville dans le Coutançais. La nomenclature se réduit encore si l'on prend en compte le "plain de la mer".

La durée du " plein " ou " plain ", c'est à dire l'étale de la pleine mer, constitue un autre facteur nautique déterminant dans le choix d'un site. En effet, plus la durée du plein s'allonge, plus une flotte de guerre dispose du temps et de la cohésion tactique nécessaire pour faire entrer ou sortir tous ses vaisseaux à marée haute dans le délai impératif d'une seule marée. Le plein de la rade de La Hougue est bref : " le havre de la hougue est facile d'entrée, long et large et l'emboucheure assez nette mais que les mariniers ne peuvent entrer que par le temps et les passes de quatre heures chaque jour d'autant que la retraitte actuelle de la mer faict assecher ledit havre ". La rade de Dieppe n'a qu'un plein limité à une demi-heure, un délai bien trop court ${ }^{62}$. En revanche, Clerville mentionne au Havre le phénomène de " tenue du plein ", aussi appelé localement le "Verhoulle ", pendant laquelle le niveau d'eau ne monte plus que très lentement et en très faible quantité durant près de trois heures ${ }^{63}$. Pourtant, cet atout exceptionnel ne profite guère au mouvement portuaire puisque l'accès du Havre est devenu si délicat vers 1664 que, d'après Duquesne, un pilote ne parvient à peine à y faire rentrer quatre terreneuviers de 12 pieds de tirant d'eau (3,96 m) durant ce temps :

"Autrefois le port du Havre estoit plus profond de beaucoup et plus commode pour les grandz vaisseaulx, ensorte qu'encore les années 1620 et 1625 des navires du Roy, la Nostre-Dame d'environ huict cens tonneaux et quatre autres de grandeur considérable demeuroient en flotte de basse marée proche de la grande Bare. Tous les moyens vaisseaux venant de Rouan et des ports voisins entroyent dans ce port facilement d'un tiers du flux. Aujourd'huy il n'y a pas d'endroict ou une barque de cinquante tonneaux puisse demeurer en flotte, et avec peine et risque les vaisseaux de Terre

61. Duquesne constate déjà en 1646 les accès restreints d'Honfleur : " A Honfleur nul vaisseau ne peut demeurer qu'il n'y prenne terre à basse marée, et pour cela il n'est point propre à de grandz vaisseaux. " (BRÉARD, Charles, " Mémoires... ", op. cit., p. 14.)

62. "Son plain ne dure que demie heure, d'autant que la rivière d'Arques qui passe au travers du havre faict retourner la mer presque aussy tost qu'elle est montée, ce qui faict que les vaisseaux ont peu de temps pour entrer au havre. " (ESMONIN, Edmond, Mémoire..., op. cit., p. 6.)

63. "Ce qui rend ce havre recommandable c'est qu'il tient son plain pendant trois heures, qui est un long temps pour donner loysir aux vaisseaux d'y entrer. " (Ibid., p. 9.) 
Neufve qui sont très moyens entrent dans ce port lors de la plaine mer, si ce n'est aux grandes marées que le vent soit propre et la mer calme ${ }^{64}$."

\section{La nature des fonds de rade et la sécurité des mouillages}

La nature du fond d'une rade constitue le troisième et dernier critère du choix d'un site portuaire. En effet, une flotte de guerre doit nécessairement mouiller sur l'ancre en rade soit en l'attente d'une marée, d'une météorologie ou d'un vent favorable pour faire mouvement, soit encore pour achever l'embarquement de vivres, munitions et artillerie. En HauteNormandie littorale, les fonds des rades sont généralement trouvés sains. Ainsi, le fond de la rade du Tréport se compose de " sable vaseux fort uni, sans roc ni écueil naturel et sans lest déchargé ". La rade est très commode à cause des abris existants et de l'ancrage qui est " parfaitement bon " pour tous navires et vaisseaux arrivés dans l'embouchure du havre " grandement sain et propre ". Voysin de La Noiraye affirme qu'à Dieppe la " rade est une des meilleures qu'on puisse trouver, ayant huict brasses d'eau et un fonds argilleux ", n'ayant d'ailleurs connu aucun changement notable depuis $1627^{65}$, une stabilité du site confirmée par l'ingénieur Gobert en décembre 1665 et Jacques de Serne, capitaine de vaisseau du roi à Dieppe. À Fécamp, Gobert estime la rade bonne, mais son port est constamment bouché par le galet ${ }^{66}$.

Voysin de La Noiraye juge la rade du Havre très médiocre pour l'ancrage : "Ce qu'il y a de deffectueux, c'est que sa rade n'est pas des meilleures pour n'avoir de basse mer que trois brasses d'eau et un fonds assez mauvais, qui est une huystrière meslée en partie de sable et en partie de roche ${ }^{67}$. " L'ingénieur Regnier Jansse le jeune déconseille tout aussi catégoriquement la rade du Havre à tous les types de vaisseaux en raison

64. BRÉARD, Charles, "Mémoires... ", op. cit., p. 6, rapport en date du $1^{\mathrm{er}}$ septembre 1665.

65. À Dieppe, les capitaines, maîtres, pilotes et mariniers affirment " n'avoir jamais veu ledit port et havre en meilleur estat qu'il n'est de présent par le moien des jettéés et espiez qui ont esté faictes a l'entrée dudit havre [...] la radde est la meilleure de toute la coste de Normandie, tant a cause du bon fondz qui est vaheux uny et esgal propre pour lever les ancres et conserver les cables, et ancres peut tenir bon contre la rigueur de toutes sortes de vents a cause de la grande quantité d'eau qui y est et que les navires sont a couvert et a labry des vents a l'oest et soroest. " (BnF, ms fr. 18596, mémoire de Nicolas Langlois de Collemoulins (1627).

66. PETER, Jean, L'arsenal..., op. cit., p. 20.

67. Langlois de Collemoulins estime déjà en 1627 la rade « fâcheuse pour estre pierreuse au fonds " et préconise les rades du Hoc et de Villerville pour la retraite des navires en cas de tempête (BnF, ms fr. 18596). La petite rade du Havre : "à la vérité, selon le rapport de quelques pilotes, elle a esté un peu gâtée par les Flamands, par les Hollandois, \& par les Anglois, lesquels soit par malice, ou autrement y ont jetté, quand leurs vaisseaux n'avoient que du l'estage, une partie de ce qu'ils portoient; de sorte qu'elle n'est pas présentement ce qu'elle valoit autrefois, quoy que les vaisseaux de trois à quatre cent tonneaux puissent seurement y moüiller, y ayant quatre à cinq brasses d'eau [la brasse à cinq pieds environ]. " (BnF, 8 LK/7 3689, de MonTEGUI, Jean-Baptiste, Mémoires et remarques sur l'importance de la ville et citadelle du Havre de Grâce..., s.l., s.d., p. 15). 
des dangers permanents menaçant la navigation, notamment les nombreux dépôts de lest abandonnés par les navires marchands :

"Cette rade a des deffaults considérables. Premièrement, elle est de très-mauvaise tenue et fort sale, tant pour les cailloux que la mer y apporte, que par le delestage des vesseaux estrangers quy y ont jetté quantité de pierres coupantes, pierres de mines et autres quy scient et coupent les cables aussy bien que par les ancres que les navires sont obligez d'y laisser. En second lieu, qu'elle n'a aulcun abry des vents de dehors quy sont ceux d'aval [d'ouest] les plus dangereux de tous. En troisiesme lieu, l'on n'y peult filler de cables faute d'espace. Enfin, on n'y peult sortir de basse mer, n'y ayant assez de fonds sur les bancs ${ }^{68}$."

L'intendant Voysin juge un peu plus favorablement Honfleur car « Sa radde est beaucoup meilleure que celle du Havre en son fonds, mais aussy elle est descouverte de tous les mauvais vents comme est celle du Havre". Son fond de vases à marée basse procure une sécurité à l'échouage aux navires venus s'y réfugier depuis la rade du Havre ${ }^{69}$. En Basse-Normandie, les informations portant sur la nature des fonds de rade ne commencent qu'à Port-en-Bessin où " la rade est fort bonne et de bon ancrage », ce que confirme un mémoire de $1746^{70}$.

La rade de Grandcamp n'autorise l'échouage qu'aux petits bateaux pêcheurs en raison de la présence du banc du Sablon. Près de Grandcamp, le Grouin de la dune dans la paroisse de Fontenay est " le lieu ordinaire où reposent les navires et bateaux venant sur les côtes de cette juridiction qui forme un grand pays plat et sablonneux propre pour l'ancrage [...] il n'y a aucune habitude, seulement le péril de la mer. Le havre du grouin de la dune à Fontenay a toujours été en l'état qu'il se trouve actuellement et est toujours le lieu de repos des bateaux venant en cette côte ", où il n'existe aucun autre lieu de repos pour les bateaux. Le port de Carentan ouvert sur la Manche par la Vire ne présente aucun intérêt pour la Marine royale car il " a ses rades plattes et il n'y peut arriver que de petits batteaux ${ }^{71}$ ". Parmi les ports et rades du Cotentin, La Hougue intéresse tout particulièrement Vauban car « la rade distante d'une portée de mousquet desdicts havres est belle pour estre de deux lieues de largeur et autant de longueur le fond de laquelle est de glèze et terre blanche sans aucune roche et de fort bonne

68. BRÉARD, Charles, "Mémoires "..., op. cit., p. 22, rapport du 31 mars 1668. Dans sa lettre du 27 décembre 1664, Regnier Jansse contredit le rapport de Duquesne qui estime Le Havre compatible avec le dessein de Colbert (DEPPING, Georges Bernard, Correspondance administrative sous le règne de Louis XIV, Paris, Imprimerie Impériale, Paris, 1854, 4 vol., t. IV, p. 30, n. 1).

69. "Et quand mesmes les navires sont agittez des grands vents a la radde du havre de grace et lieux circonvoisins le plus souvent ils sont contrainctz pour leur seureté de venir eschouer sur les vazes de ce lieu " (SHD Vincennes, SH 48).

70. " Port en Bessin est un havre fort commode et Sa Majesté a donné quelques ordres de le rétablir " (GouHIER, Pierre, "Port-en-Bessin (1597-1792). Étude d'histoire démographique ", Cahier des Annales de Normandie, n ${ }^{\circ}$ 1, 1962, p. 15 et Id., L'Intendance..., op. cit., p. 133).

71. GOUHIER, Pierre, L'Intendance..., op. cit., p. 225. 
teneure $^{72}$ ». En revanche, Barfleur n'a qu'un accès limité par un banc de sable à l'entrée du havre :

" [H]aussé de viron deux pieds tant par le sable que la mer y a par sa violence apporté de dehors que par celuy qui sy est déroulé du fonds du havre par le moyen d'un fort courant qui roule par le milieu dudit havre et que le banc s'est avancé dans ledit havre de la longueur de viron deux toises, que le fonds et assiette dudit havre est dur et ferme en quelques places et plus molle en l'accès y ayant deux petits rochers dans le milieu iceluy nommé la branche et petite poignante élevés du fonds de la hauteur d'un pied et demy ou viron."

Le havre de Cherbourg est établi du côté de l'ouest contre la ville, château et faubourg de Cherbourg, et du côté de l'est contre les sables et mielles (dans le patois de la Manche, ce sont des grèves plates, sèches et mobiles), sans causer aucun préjudice à l'embouchure du havre : " ledit havre est de present meilleur quil ay testé y ayant esté faict une petite jettée des deux bords de l'emboucheure dudict havre qui cause par la rapidité de l'eau que ledict havre soit apronfondy ". mais aucun commentaire des comparants n'insiste sur la piètre qualité du fond de la rade pour l'ancrage des navires. Enfin, plusieurs procès-verbaux livrent ponctuellement quelques informations complémentaires sur les sites portuaires.

\section{Les informations complémentaires retirées des procès-verbaux}

\section{La question de l'évolution du trait de côte}

Plusieurs officiers d'amirauté mentionnent l'intéressant problème du changement du trait de côte ou du profil de rivière ${ }^{73}$. Ainsi, le phénomène qui apparaît le plus fréquemment sur le littoral normand est celui du comblement des ports et havres, soit par les vases et galets, soit par les bancs de sable. En Haute-Normandie, le recul de la falaise et la formation d'un stock de galets déplacé par les courants et les vagues est à l'origine de l'accumulation de ce matériau dans les ports du Pays de Caux ${ }^{74}$. Il se crée au Tréport des "perroys" (perreys) de galets accumulés au sud de ce port. Le remède serait, selon les comparants, de rallonger la jetée bâtie du côté du sud pour bloquer la progression du galet. Les principaux marchands notables de Dieppe (Chauvel, Néel, Faulcon, Dupuis, Le Griel et Locquin) déclarent, après une longue conférence entre eux, " qu'ils ne peuvent parler de la qualité de la rade de cette ville pour nestre de leur compétence que

72. L'intendant de Caen Foucault confirme la qualité de la rade de La Hougue (GouHIER, Pierre, Ibid., p. 132, n. 140). Sur la description (avec cartes) de la rade de la Hougue : EsSAR, Dennis F. et PERnAL, Andrew B., "Le Vasseur de Beauplan et les installations portuaires en Normandie et en Bretagne au XVII ${ }^{\mathrm{e}}$ siècle : une lettre inédite à Jean-Baptiste Colbert ", Annales de Normandie, 43e année, n 1, 1993, "Rivages de Normandie ", p. 41-59.

73. Sur la définition, paradoxalement délicate, du trait de côte, voir SALOMON, Jean-Noël, Géomorphologie sous-marine et littorale, Presses universitaires de Bordeaux, 2009, p. 94-95.

74. Musset, René, "La côte du pays de Caux ", Annales de Normandie, ${ }^{\text {re }}$ année, $\mathrm{n}^{\circ} 2$, 1951, p. 110-117. 
le port et havre en est a present passablement bon, mais en tout temps sujet au changement ce qui peut estre corrigé par le moyen des jettées ".

Au Havre, le recul du cap de la Hève consécutif aux éboulements de la falaise (en 1666, recul de plus de 50 toises, soit 100 mètres environ en trente ans d'après les habitants du Havre) alimente régulièrement le mouvement du galet tant vers la pointe du Hoc en Seine que vers le Hâble d'Ault à proximité de l'estuaire de la Somme ${ }^{75}$. Les plus anciens et experts bourgeois de Honfleur confirment que « le plus souvent les raddes sont plus profondes y ayant long temps quelles ne l'avoient esté sy peu Ce qui provient de ce que ledit canal ne se descend point a droicte ligne en ces raddes comme il faisoit au temps passé "; ils ajoutent que le canal ou ruel d'Honfleur contient " plus de 20 pieds d'eau contre 30 pieds autrefois lorsqu'il estoit en son premier estat ${ }^{76}$ ». Les 150 pilotes de l'amirauté de Caudebec " sont obligez de lune en lune de sonder les lieux dangereux et de voir le diep ou canal de la rivière à cause des bancs de sables muables qui se rencontrent et qui font changer le diep ou chemin de marée ". Les pilotes attestent qu'ils ont trouvé ou trouvent encore de présent dans le canal de la Seine depuis la rade du Havre ou de Honfleur jusqu'à Quillebeuf

" qu'il n'y a audit Quillebeuf qu'une rade ou posée où les bâtiments s'échouent à toutes les marées en attendant la marée propre pour monter ou descendre, qu'elle a été toujours et est encore très bonne pour mettre à l'abri jusqu'à 150 vaisseaux des vents d'ouest à sud-ouest, sud et sud-est ${ }^{77}$ ".

En Basse-Normandie, les mouvements des marées vont jusqu'à affecter la navigation fluviale. La visite de l'Orne du 10 avril 1665 emprunte la partie aval du fleuve, de Colombelles à Longueval. Les pilotes affirment

" qu'il y a cinquante ans et encor depuis quilz ont veu la riviere profonde de plus de dix pieds deau de plaine mer parce que la riviere estoit plus estroite de la moityé pourquoy les batteaux et navires de grandeur de Cent thonneaux et plus alloient pour lors facillement a la ville de Caen chargez ou a present Il ny peut aller que du port de quarante a cinquante thonneaux et encore de quinzaine en quinzaine et durant la grande mer et que Sil ny est remedyé ladicte riviere ne pourra plus estre navigable ".

Selon eux, l'élargissement du cours de la rivière et la diminution importante de sa profondeur s'expliqueraient " par le flux et reflux de la mer et Inondations et débordements deaux qui ont remply le cours faute d'entretien et faute de digues tallutz et pieux le long des bords dicelle. " En

75. Duquesne constate que « le cap de la Hève se ronge et se mange continuellement par la mer et donne passage aux cailloux. " (PETER, Jean, L'arsenal..., op. cit., p. 17).

76. C'est exagéré : Langlois de Collemoulins note en 1627 que si des navires de 500 tx y entrent très facilement, le havre d'Honfleur n'a que 18-20 pieds d'eau de profondeur de grande mer. En revanche, il juge le havre d'Honfleur " tres bon, tres seure, et de facille abord [...] ses jettées y sont tres bien entretenües et en bon estat et les vaisseaux qui y abordent y sont en toute seureté " et signale le détournement de la petite rivière d'Honfleur hors de son canal habituel " par les ruynes que la mer y a faictes " (BnF, ms fr. $\mathrm{n}^{\circ}$ 18596).

77. BRÉARD, Charles, " Mémoires... ", op. cit., p. 6. 
revanche, les rivières du pays du Plain dans le ressort de l'amirauté de Carentan ne connaissent guère d'évolution, car l'accumulation de sédiments marins demeure constamment positive dans les estuaires de la baie des Veys ${ }^{78}$. Les comparants du lieu déclarent d'ailleurs que, dès leur plus jeune âge, " ils ont vu ces rivières et havres dans le mesme estat qu'actuellement sauf lors des inondations et impétuosité des eaux l'hiver, et la violence des grands flots et marées".

Le phénomène d'évolution du trait de côte se manifeste régulièrement près de l'estuaire de l'Orne, mais sans modification irréversible dans les petits havres du Coutançais et du Cotentin. Sur le littoral, le havre de Grandcamp se trouvait autrefois dans un lieu plus éloigné que l'actuel et joignait la paroisse de Criqueville; il est à présent hors d'état pour y abriter des bateaux : l'embouchure de la rivière du Verrey (le Véret) qui se jette dans ce havre a été remplie par les " apports de la mer " (pierres et sablon), ce qui empêche son " dégorgement " par le rehaussement sédimentaire. Les comparants jugent que 30000 lt au moins seraient nécessaires pour remettre l'ancien havre en état, somme trop élevée et non justifiée pour un si faible trafic. Le même inconvénient se produit à Vierville, distant d'une lieue et demie de Grandcamp. Ce petit havre situé dans un abaissement de la côte n'accueille plus de bateaux depuis plus de trente ans parce qu'il a été rempli de sablon " par l'impétuosité de la mer"79".

Dans le Cotentin, « le havre de Quinéville est fort enfoncé dans les terres et l'entrée assez difficile tant a cause des bancs faisant les deux costés d'une estroitte et pettitte riviere partant dudit havre pour aller a la mer, qu'a raison du peu d'eau qui demeure sur lesdicts bancs ". Au havre de Regnéville, "la force desdites marées change quelque foys le cours de ladite rivière et fait former quelques bancs de sable par aucunes foys proche de ladite rivière, et aucunes foys elle les destruicts et ruinent par son montant naturel $^{80}$ ". Germain et Pierre Tanquerey de Coutainville déposent qu'ils ont

" tousiours veu ledit havre en mesme estat et fort difficile et périlleux dans son entrée a cause des bancqs de sable qui sont a l'emboucheure d'icelluy et qui se changent au gré des vents et de la mer pourquoy ont esté obligés de planter en ladite entrée des marques et balizes lorsque le cour d'un

78. Le bilan sédimentaire de chaque marée du lieu est inexorablement positif en raison de l'énergie du jusant inférieure à celle du flot (BournÉRIAS, Charles et al., La Manche du Havre..., op. cit., p. 28-29). La baie des Veys a toujours eu la même largeur selon les comparants : " une lieue de large au port de Létenière, un quart de lieue entre Brévent et Burcheille, 40 perches au Four de Taute. La marée monte au Four de Taute et dans la baie et rivières de 12-15 pieds de haut au haut de l'eau. " (SHD Vincennes, SH 48.)

79. Langlois de Collemoulins note en 1627 que le havre de Vierville " a présent est inutilles pour estre remply de sable" (BnF, ms fr. 18596).

80. Sur la dérive littorale nord-sud qui reprend une partie des sables d'origine éolienne datant du Holocène et les sédimente à l'embouchure des petits cours d'eau côtiers en formant une flèche littorale orientée nord-sud, contraignant la rivière à décrire un $\mathrm{S}$ pour gagner la mer en formant un havre, voir BOURNÉRIAS, Charles et alii, La Manche..., op. cit., p. 28. À Portbail en 1627, "ledit havre est de difficille entrée a raison de plusieurs sinuositéz qui y sont " (BnF, ms fr. $\left.\mathrm{n}^{\circ} 18596\right)$. 
petit ruisseau qui court dans ledit havre qui est un havre de barre se trouve diverty tellement que dans le plain des marées communes et hors le temps de la morte eau Il ne peult entrer dans ledit havre que des vaisseaux du port de environ vingt tonneaux ny montant davantage que environ huict pieds d'eau durant trois a quatre jours par chaque quinzaine".

L'ensablement progressif des havres du Cotentin occidental par la formation de barres à l'embouchure de l'estuaire entraîne la diminution régulière des tonnages fréquentant ces parages ${ }^{81}$. Ce phénomène explique aussi en grande partie la disparition des armements terre-neuviens locaux au $\mathrm{XVII}^{\mathrm{e}}$ siècle $^{82}$. Au havre de Lingreville, Barnabé Hüe, âgé de 46 ans, matelot et propriétaire d'un bateau naviguant depuis ce havre, " a cognoissance que depuis plus de vingt cinq ans ledit havre n'a aucunement changé [...] mais que auparavant ledit havre estoit fort bon et fort facille comme il l'a appris par ses ancêstres sy ledit havre n'esté gastey par des bancqs de sable qui se sont former a l'emboucheure et a l'entrée dudit havre a cause de l'impétuosité du vent et de la mer ". Au havre de Pirou comme dans celui de Saint-Germain ${ }^{83}$, les comparants signalent le changement très fréquent de l'entrée à cause de la présence de bancs de sables.

\section{La lutte contre le délestage anarchique des navires}

Les autorités luttent également contre le délestage anarchique des vaisseaux dans les ports et havres. En effet, le lest d'un navire est composé de pierre ou de sable entreposé à fond de cale. Cet élément de stabilité du navire, surtout lorsque le bâtiment est lège, est indispensable à sa sécurité lors d'une navigation. En revanche, sa masse peut éventuellement déformer, voire crever, la coque du bâtiment pendant un échouage ${ }^{84}$. Les capitaines et maîtres de navires doivent donc délester leur navire à l'arrivée en déposant le ballast dans un lieu désigné et marqué par les officiers du

81. Langlois de Collemoulins constate que l'embouchure de l'Orne à Estrehan en 1627 est " de tres difficille abord pour les longs bancs de sables \& grandes battures qui sont à l'embouchure [...] et que le flux de la mer change quazy a toutes les maréés " (BnF, ms fr. $\mathrm{n}^{\circ}$ 18596).

82. LA MORANDIÈRE, Charles de, Histoire de la pêche française de la morue dans l'Amérique septentrionale, Paris, éd. Maisonneuve \& Larose, 1962, 3 vol., t. I, p. 239 et 287.

83. " Les comparants ont dit qu'ils ont veu ledit havre de Saint Germain en mesme estat dans lequel il pouroit se retirer quantité de navires a cause de sa longueur quand mesme lesdits navires seroient du port de cent thonneaux et qui pourroient entrer dans ledit havre en cognoissant l'ouverture et lentrée d'iceluy qui est aucunes foys changée par quelques bancqs de sable qui divertissent le courant de la rivière qui vient du dedans les terres, que l'ouverture dudit havre est quelquefoys contigüe de lune des deux roches qui sont a l'entrée diceluy qui pourroit rendre ladite entrée difficile [...] " (SHD Vincennes, SH 48.)

84. Arch. mun. du Havre, fds. anc., BB 4. C'est pour cette raison que l'interprète en langue basque Jean Deneufville et Pierre Martin, interprète des langues flamandes sont condamnés par l'échevinage havrais à cause d'un navire (baleinier?) qui a fait son lestage à marée basse, au risque de crever sa carène et d'encombrer le port au retour du flot, 30 août 1642 . 
siège d'amirauté local. Le problème demeure secondaire dans les petits ports et havres du Cotentin car bien souvent l'équipage se charge seul du délestage. À Linverville, Gonneville et Gouville, "lorsqu'il arrive des batteaux dans lesdittes raddes qui apportent leurs lests, les mathelots le font a mesme temps transporter au plain de la mer vis-à-vis et a l'endroit des raddes dont les habitants se servent à faire des maisons". À Lingreville et Bricqueville, aucun lest n'a été versé dans le havre depuis plus de vingt ans car les petits bateaux du lieu jaugeant au plus trois tonneaux n'emploient aucun lest à bord.

À Port-en-Bessin et à Sainte-Honorine, les bateaux sont halés à terre au cabestan ou " hauvers en barre ". Le lest du bateau est " mis hors et amonté " au-delà de l'estran pour que cela n'apporte aucun dommage aux bâtiments suivants. La décharge des lests à Carentan se fait contre les " diqueries " des rivières pour les renforcer contre les inondations de la Vire et de ses affluents ou pour la réparation des chemins ${ }^{85}$. Aucun dépôt de lest n'y est non plus remarqué sauf sur la chaussée submersible de Saint-Fromond gênant le passage des navires. En revanche, à Barfleur, le mauvais état du

" fonds du havre ou posent ordinairement les barques et navires est bordé de plusieurs rochers sur et au-dessus desquels nous ont dit avoir de tout temps vu jeter et délester les lests des vaisseaux lesquels descharges ainsy que les immondices qui procedent du parcage des huistres que l'on parque ordinairement sur les bords dudit havre ont fait hausser le fond dudit havre de viron un pied et les bords de deux ou trois".

Les réactions des autorités locales varient surtout selon la nature de la gêne causée au public et à l'environnement naturel immédiat. Elles n'atteignent pourtant jamais le niveau d'inquiétude élevé des quelques observateurs du siècle suivant dénonçant "l'infection des mers et l'empoisonnement des côtes ", à l'origine de la diminution de la " fécondité des océans $^{86}$ ".

Le problème du lest diffère nettement dans les ports marchands où le trafic n'a rien de commun avec celui des petits havres. Les officiers et maîtres de quais surveillent constamment le délestage des navires et le dépôt de leurs lests au lieu indiqué selon sa nature. Les vaisseaux qui entrent dans Cherbourg ont un lest composé en partie de sable et en partie de galet, mais les conditions naturelles du port impliquent une séparation des sites de stockage. Le sable se décharge à l'est du havre et le galet du

85. Nouveau glossaire nautique d'Augustin Jal, lettres D-E, Paris, CNRS, 1983, p. 456, action de construire des digues. BARRÉ, Éric, "À propos de digues, position d'un problème à partir de documents inédits concernant la baie de l'Ouve aux XIV et Xve siècles ", actes du $47^{\mathrm{e}}$ congrès des Sociétés historiques et archéologiques de Normandie (SHAN), Bernay, 17-18 octobre 2012, Eaux vives, eaux dormantes en Normandie, éd. Fédération des SHAN, $\mathrm{n}^{\circ} 18,2013$, p. $71-77$.

86. " Les pêcheurs eux-mêmes [...] ont pour habitude de rejeter déchets et immondices sur les huîtrières qu'ils infectent " (CORBIN, Alain, Le territoire..., op. cit., p. 230). 
côté de l'ouest hors du havre, tant pour que les vaisseaux qui ont du lest à prendre puissent s'en lester que pour éviter le refoulement des galets dans la rade par les courants. Les navires délestent aussi avant de remonter un cours d'eau pour réduire le tirant d'eau. Les anciens maîtres interrogés n'ont jamais vu décharger aucun lest dans le havre de Ouistreham " qui a son emboucheure de la mer qui a son flux et reflux dans la rivière d'Orne qui va du havre de Ouistreham dans la riviere de Caen et qu'au contraire les navires qui viennent de la rivière de Caen se lestent de sable qui est audit havre ". Sur la Seine, à Quillebeuf et autres posées du ressort de cette juridiction, "les navires ne lestent ni délestent que fort peu. Lorsque cela se fait par quelques-uns des maîtres de navires, les officiers font mettre le lestage au haut de la pleine mer et proche de la côte. " Parfois, les maîtres de vaisseaux n'ont pour seul lest en remontant la Seine vers Rouen que leur chargement. Par exemple, les heux de Picardie chargés de sablon revendent ce lest aux habitants de Rouen ${ }^{87}$; au retour, ils appareillent lège depuis Rouen avant de charger du fret aux carrières de Dieppedalle ou de Caumont (pierres de taille).

On reste plus dubitatif à la lecture d'autres procès-verbaux. Peut-on croire sur parole les capitaines, maîtres et bourgeois d'Honfleur affirmant qu'aucun navire français ou étranger ne décharge de lest dans le canal ou en rade parce qu'il " n'y a aucun lieu destiné au dépôt du lest puisque les navires n'en apportent pas dans le port "? Au Havre, Voysin de La Noiraye reste prudemment évasif dans son rapport : "On dit que cette rade a estée gastée par le lest des vaisseaux que l'on y a deschargé ", car ce genre d'affaires relève du gouverneur et de l'échevinage ${ }^{88}$. Il faut en fait y voir comme très souvent une histoire de " gros sous ". Dans l'enquête des amirautés de 1665, aucun procès-verbal ne signale l'existence permanente de droit de lestage ou de délestage de navires en Normandie, à l'exception de celui du lieutenant du siège de Rouen, du 23 avril 1663 confirmé en octobre 1664, le seul de la province à mentionner scrupuleusement le règlement de l'amiral, le duc de Vendôme.

\section{La dégradation inquiétante de l'outil portuaire en Normandie}

Comme on l'a vu, les ports normands sont tous des havres de marée, asséchant à marée descendante. La plupart d'entre eux sont, de surcroît, naturellement menacés d'envasement et de comblement de leurs accès par le galet ou le « sablon ». Le port de Fécamp " où il n'y a que des bateaux de

87. Le heu est un navire à fond plat utilisé pour le trafic maritime dans la mer du Nord et la Manche (Nouveau glossaire nautique d'Augustin Jal, lettre H, Paris, éd. CNRS, 1992, p. 902-903).

88. Esmonin, Edmond, Mémoire..., op. cit., p. 9. Le maître de quai du Havre est responsable du lestage et délestage des navires. Sa nomination revient au gouverneur de la ville avec droit de regard de l'échevinage (DARSEL, Joachim, "L'Amirauté en Normandie. IV. Amirauté du Havre-Harfleur ( ${ }^{\text {re }}$ partie) ", Annales de Normandie, $20^{\mathrm{e}}$ année, $\mathrm{n}^{\circ} 4,1970$, p. 286. 
pêcheurs est rempli de galets ". Duquesne mesure l'ampleur de l'envasement portuaire au Havre entre 1620 et $1665^{89}$. Si les phénomènes naturels expliquent largement les accès des ports normands rendus difficiles, il faut aussi imputer aux négligences humaines une bonne part de responsabilité dans le délabrement général des infrastructures portuaires.

Les infrastructures d'un port nécessitent un entretien régulier pour rester opérationnelles, mais cela n'est pas toujours compatible avec des finances publiques ou municipales fragilisées par la guerre, comme la banqueroute de l'État en 1648, par exemple. Le quai de Rouen, le cœur marchand de la place avec la Bourse des marchands ${ }^{90}$, en constitue un exemple particulièrement significatif ${ }^{91}$. En dépit du flot continu de passagers et de marchandises ${ }^{92}$, les périodes d'entretien des quais alternent avec des phases d'abandon. Ainsi, de nombreuses portions du quai font encore l'objet d'améliorations successives au début du XVII ${ }^{\mathrm{e}}$ siècle : le quay des navires est taluté en 1608, le quay de la voiture d'Elleboeuf est pavé, "piloté " (consolidé verticalement par des "piles " ou pilotis plantés en rive) et taluté en 1616, le quay de Paris est à son tour taluté et pavé en 1617, suivi par le quay du plastre en 1638. Rien ensuite, jusqu'en 1660 où l'on bâtit le nouveau quay aux pierres. La longue durée des négligences d'entretien et de curage des abords des quais perturbe pourtant le mouvement portuaire d'après le procès-verbal du lieutenant de l'amirauté de Rouen Duhoulley : "Les taluts depuis la porte Guillaume Lion jusqu'à la porte Saint Eloy sont en quelques endroits gastez tant par les vidanges des escalles que des fenges qui donnent en quelque façon empeschement aux vaisseaux qui sont chargez d'approcher facilement des quais lesquelles fenges ne proviennent que du coulland que la rivière apporte. "

Les chemins de halage longeant les rives de la Seine sont eux aussi trouvés en mauvais état entre Rouen et l'aval jusqu'à La Mailleraye ${ }^{93}$. La

89. "À l'époque de Richelieu, Le Havre accueillait des vaisseaux de huit cents tonneaux alors qu'en 1665 ceux de cinquante s'y conservent avec beaucoup de peine et de risque [en dehors des grandes marées]. " (Vergé-Franceschi, Michel, Abraham Duquesne..., op. cit., p. 206.)

90. Le voyageur anglais John Clenche note en 1675 que le commerce de Rouen possède alors " an ill-favoured Bourse, hung with ugly Pictures of their French kings, a small Trade with all sorts of Merchandize, but the most considerable commodity is English Lead " (Lough, John, France observed in the Seventeenth Century by British Travellers, Stockfield, Oriel Press, 1985, p. 60).

91. FARIN, Histoire de la Ville de Roüen..., op. cit., p. 374-375.

92. Ellis Veryard remarque en 1701 que la Seine est pour le commerce de Paris : "its chief support, serving to import and export all sorts of Merchandize, and giving it all the advantages of other Cities that lie on the main Ocean; for this River is navigable from Rouen hither, and from hence divers Leagues up the Country, at least for large Boats and Barges. " (Lough, John, France..., op. cit., p 56.)

93. "Ont vu que le long du rivage où il y a halage les chemins sont très mauvais et gastez en beaucoup d'endroits ce qui donne beaucoup de peine aux navires qui montent la rivière depuis la Mailleraye jusqu'à Rouen ce qui rend la navigation plus difficile et lorsque les navires remontent la rivière avec les vents contraires ou quand il court un 
navigation sur le fleuve se heurte également aux dangers des bancs mouvants de sable et de vase, imposant le recours aux pilotes :

"La rivière de Seyne est fort large en son entrée, et jusques a Quillebeuf est plaine de bancs de sable mouvant; depuis le Havre jusques a Honnefleur, et d'Honnefleur jusques a Quillebeuf elle est aussy subjecte a un flot impétueux qu'on apelle la Barre. Quilboeuf est un lieu très important, tous les vaisseaux qui viennent de la mer estans obligés d'y arrester parce qu'une marée ne les peut porter, et de plus il y a vis-a-vis un banc de sable changeant pour lequel éviter on est obligé de prendre des matelots du lieu ${ }^{94}$."

La dégradation des installations portuaires, ou à tout le moins leur vétusté, est tout aussi préoccupante sur le littoral cauchois. Le défaut de déblaiement des vases du port et des affaissements de terrains provenant du ruissellement des eaux pluviales expliquent le comblement progressif du havre et du " canal " (chenal) de Saint-Valéry-en-Caux depuis la visite en 1627 de Langlois de Collemoulins ${ }^{95}$. Le procureur du roi souligne le poids de la guerre maritime dans le délabrement des installations portuaires, mais pointe aussi la négligence des lesteurs de navires ${ }^{96}$. Dans le Cotentin, l'embouchure du havre de Cherbourg est menacé par le risque d'effondrement du pont de pierre ${ }^{97}$. Les conflits d'usage ne sont pas rares entre voies de communications : la descente de la Vire en barque depuis Saint-Fromond jusqu'à l'embouchure du Petit Vey est gênée entre Montmartin et le marais de Higate par un apport et amas de grandes quantités de pierres servant de chaussée submersible et passage au lieu appelé Raye Guérout.

La situation du Havre apparaît autrement plus critique, tant en raison de son importance commerciale et stratégique, que par l'ampleur du déla-

grand esbe en temps calme il faut que les vaisseaux soient tirés par des chevaux. " (SHD Vincennes, SH 48.)

94. Esmonin, Edmond, Mémoire..., op. cit., p. 10. Selon Thomas Corneille, « Le passage du Havre à Quillebeuf est en réputation d'être difficile, à cause de la quantité de bancs de sable qui s'y forment et qui changent de place, ce qui oblige les vaisseaux étrangers à prendre des pilotes de Quillebeuf " (Dictionnaire (1702), art. Quillebeuf).

95. Le port de Saint-Valéry est " très bon et en bon estat par le moien des ouvrages et jettées qui ont esté faictes depuis douze a treize ans en ça... "; à Fécamp, Langlois de Collemoulins trouve le port et havre " en fort mauvais estat et presque bouché ", 1627 (BnF, ms fr. 18596).

96. "Le malheur qui a causé l'empirance dicelluy ne provenant que de la perte des vaisseaux depuis Vingt cinq ans par la ruinne des guerres ce qui a reduict les habitants dans Limpuissance den pouvoir rebastir nestant besoing de grande despense pour la reparation d'Icelluy \& leur Lesteurs qui empeschent Louverture des eaux qui pourroient couller au dessus des escluses ainsy quils faisoient par Le passé \& Ensuitte repousser le perroy qui arrivé au bout des pallissades par limpétuosité de la mer... " (BnF, Mél. Colb., 128 bis. Rigoult à Colbert, lettre du $1^{\mathrm{er}}$ mai 1665.)

97. Ce havre qui est "établi Nort et su, allant depuis ladite emboucheure jusqu'à un pont de pierre contenant dix arches soubz lequel il passe la petite rivière qui reste dans ledict havre de basse mer et y donne son cours pour vuider laditte emboucheure duquel pont il est écroulé depuis peu deux arches. Le reste menaceant Ensemble ruyne par sa vieillesse et antiquité dont la cheuste pourroit faire détourner le cours de laditte riviere de l'emboucheure dudict havre ce qui en causeroit la ruine... " (SHD Vincennes, SH 48). 
brement des infrastructures portuaires. Cette situation résulte tout d'abord des erreurs d'appréciations commises par l'architecte Regnier Jansse l'aîné dès la construction du bassin à flot en $1635^{98}$. Les installations portuaires ont ensuite été entretenues vaille que vaille jusqu'à la fin du ministériat de Richelieu $^{99}$. Mais une décennie plus tard, « tout menace prompte et entière ruine " : les jouyères de maçonnerie, le pont levant double de l'écluse, le talus écroulé sont à l'abandon faute de fonds. En mars 1657, le bassin à flot est totalement impraticable aux navires. Le bassin est presque entièrement comblé de vase. Les portes rompues de l'écluse ne retiennent plus l'eau à marée descendante. Le ponton et " pipery " pour caréner les navires sont détruits. Le port part à vau l'eau, mais les échevins havrais n'obtiennent aucun financement de la duchesse d'Aiguillon ${ }^{100}$. De plus, la tempête cyclonique du 22-23 novembre 1662 endommage le port déjà fort mal en point et presque obstrué de graviers et galets à l'entrée ${ }^{101}$. Ainsi, la dégradation plus que préoccupante des infrastructures portuaires du Havre jointe à la question essentielle de ses accès pour les navires de fort tonnage n'exige pas moins de trois études successives analysées par Jean Peter ${ }^{102}$.

L'exemple du Havre souligne plus généralement la qualité discutable des matériaux employés dans les travaux de réparations portuaires ${ }^{103}$, le manque criant de moyens financiers ainsi que la très forte pression fiscale ressentie durant les conflits de la période 1635-1659. À Barfleur, aucun fonds n'est " employé pour la répurgation dudit havre ». L'entretien du canal

98. Vergé-Franceschi, Michel, Abraham Duquesne..., op. cit., p. 206.

99. La défectuosité du système hydraulique des barres de chasses a toujours constitué le talon d'Achille du port havrais sous l'Ancien Régime. Langlois de Collemoulins conseillait déjà en 1627 d'améliorer le système défectueux des barres et écluses par de nouvelles plates-formes élargies afin de lutter contre l'envasement du port (BnF, ms fr. $\mathrm{n}^{\circ}$ 18596).

100. Papillon, échevin du Havre, a été reçu par la duchesse, qui tient le gouvernement du Havre, en présence de Denis Marin (le futur secrétaire particulier de Colbert en 1661) et de Gargan, intendant de finances, mais aucune somme ne peut être allouée au rétablissement du bassin, du pont et des barres, 3 mars 1653 (Arch. mun. du Havre, fds. anc., BB 6).

101. Peter, Jean, L'arsenal..., op. cit., p. 1-2 et 15-16. Esmonin, Edmond (éd.), Mémoire..., op. cit., p. 8-9. Duquesne informe Colbert que « le port est comblé de vases; les épis [édifiés perpendiculairement au trait de côte sur l'estran pour bloquer la course du galet] n'ont pas été entretenus; les barres ne courent plus que 12 fois par an depuis plusieurs années au lieu de 60 autrefois; le pionnage, l'enlèvement du galet et de la vase ne se font pas assez régulièrement" ".

102. Études des travaux d'urgence et devis confiés successivement à Nicolas Madiot, Guillaume Métezeau et François Blondel entre le 30 décembre 1662 et le 10 décembre 1664 (PETER, Jean, op. cit., p. 5 et 16).

103. Rapport final de la chevauchée de Colbert du Terron du 15 mai 1665 : «En tous les endroicts maritimes que j'ay visités en ce voyage, j'ay remarqué que les ouvrages des anciens, pour la commodité de la navigation, estoient faicts avec plus de prévoyance et des matériaux plus solides qu'aujourd'huy. Je suis honteux qu'en nos derniers temps il paroisse tant de timidité, et qu'il y ait si peu de gens qui se soit intéressez au restablissement des ouvrages concernantz la marine tout-à-fait importante pour la gloire de l'Estat. » (BRÉARD, Charles, "Mémoires... ", op. cit., p. 20.) 
ou " ruel " d'Honfleur menant au bassin consiste, d'après les bourgeois et experts du lieu, en pose de fascines de brières et de longs pieux de bois. Le financement de ces opérations provenait des don et octroi du droit de quatrième concédé par le roi aux habitants de la ville. Mais le roi ayant repris quelques années auparavant la moitié du provenu de ce droit, les habitants ont saisi au vol ce prétexte pour ne plus payer les travaux de maintenance. Faute "d'entretenement ", le cours du canal s'est modifié " et passe de présent a cause de la mutation du courant de la rivière de Seyne par un lieu dont l'entrée est plus difficile que auparavant ${ }^{104}$ ".

Les négligences constantes des usagers des ports provoquent également l'obstruction partielle des installations. Les fossés d'Honfleur, saturés de vases faute d'entretien, ne remplissent plus leur fonction de chasse pour maintenir une profondeur suffisante dans le port ${ }^{105}$. En dépit du curage du bassin de Dieppe entrepris par l'échevinage depuis 1657, Saint-Victor, le lieutenant du siège note que

" pour le regard dudit havre, quil est necessaire de le faire curer attendu quil est remply de pierres et immondices qui causent que l'on ne peut trouver en iceluy aucuns plats ou les vaisseaux puissent estre en seureté, ny demeurer en flotte de basse eau, comme on les a veu cy devant jusques a trois et quatre en haye ou a costé les uns des autres".

Mais ni le lieutenant du siège, ni les marchands du lieu n'osent révéler ouvertement les errements comptables commis par les fermiers du droit de quayage de Dieppe dont les fonds devraient, en principe, être affectés à l'entretien des quais ${ }^{106}$.

104. « Sy le grand canal par lequel Il fault entrer dans cedit port change de fois a autres cela provient du manque dentretien d'icelluy avec des pieux et facinnes [...] ce qui a causé la rupture des quais des jettées et du bassin de ce dit lieu qui dépérissent journellement. " (SHD Vincennes, SH 48.)

105. « Il faudroit par advance faire travailler incessamment à la vide des vases et immondices qui remplissent et comblent les fossez de la ville, ce qui est cause que l'eau des bares ou escluses n'ont plus de force pour entretenir les portz ouvertz à quoy autrefois elles ont esté destinées. " (BRÉARD, Charles, "Mémoires... ", op. cit., p. 20.)

106. Seul Clerville souligne " le peu de soin qu'ont eu les administrateurs des droits de quayage, d'employer à son entretenement les deniers qu'ils ont cy devant receus pour cette fin, de sorte que les choses y sont en un si déplorable estat " (BnF, Cinq Cents Colbert, $\mathrm{ms} \mathrm{n}^{\circ}$ 122). Lettre de Saint-Victor, lieutenant de l'amirauté de Dieppe à Colbert sur les " petits abus et mauvais mesnages de deniers destinés pour les ouvrages et travaux de notre dit quaÿ et auxdites adiudications qui se font dans la maison de ville tant de la présente ferme du quaÿ que travaux et ouvrages, [...] et l'on n'entendroit point parler non plus de ce qui se passa une fois dans la maison de ville en la personne d'un vieil eschevin fort homme d'honneur lequel lors des dittes adiudications, sommeillant fust réveillé par un des aultres conseillers qui luÿ dit de donner son attention aux expéditions qui se faisoient, Il luÿ fist donner response quil estoit dans un songe qui l'avoit tenu long temps en grand embarras, et qu'il croÿoit voir des loups de mer qui rongeoient les pallissades et jettéés de notre port et havre. Ce quon a voulu expliquer de messieurs ses confrères qui disposoient a leur profict particulier des deniers qui debvoient estre fidellement emploÿéés auxdittes pallissades et jettéés, ces loups de mer ont duré en apparence jusques a présent, veu l'estat ou sont les choses, et dureront si votre justice et votre authorité n'interviennent..." (SHD Vincennes, SH 48.) 
En Basse-Normandie, le cours de l'Orne entre Caen et l'estuaire représente presque un cas d'école par l'importance des dégradations, conflits d'usage et malversations concentrés dans le procès-verbal de 1665. Cette importante voie d'échange fluviale entre Caen et la Manche subit régulièrement pendant la mauvaise saison des "crétines " ou crues provoquées par la rencontre du flux de la mer à marée montante avec l'Orne au niveau du pont Frileux. La situation empire par l'absence de tout entretien du cours de ce cours d'eau depuis deux à trois décennies ${ }^{107}$, si bien que les éboulis des talus et murailles de la ville emplissent la rivière au droit des rives et gênent les poses des bateaux à la basse $\operatorname{mer}^{108}$. De ce fait, sa profondeur a considérablement diminué par endroit, comme le montre le tableau suivant.

\section{Tableau 5 - Variations de la profondeur de l'Orne à Caen}

\begin{tabular}{|c|c|c|c|c|}
\hline $\mathrm{N}^{\circ}$ & $\begin{array}{c}\text { Secteur de la rivière } \\
\text { (sa distance en perches à } \\
24 \text { pieds la perche) }\end{array}$ & $\begin{array}{c}\text { Hauteur d'eau à } \\
\text { pleine mer (en } \\
\text { pieds) }\end{array}$ & $\begin{array}{l}\text { Hauteur d'eau } \\
\text { à basse mer de } \\
\text { morte eau } \\
\text { (en pieds) }\end{array}$ & $\begin{array}{l}\text { Largeur de la } \\
\text { rivière }\end{array}$ \\
\hline 1 & $\begin{array}{l}\text { Pont de bois-Foire royale/pont } \\
\text { St Jacques ( } 15 \text { perches) }\end{array}$ & $9-10$ & 5-6 & $40-50$ pieds \\
\hline 2 & $\begin{array}{l}\text { Pont St Jacques/grosse tour } \\
\text { de ville }\end{array}$ & $9-10$ & $5-6$ & $40-50$ pieds \\
\hline 3 & $\begin{array}{l}\text { Grosse tour de ville/moulin } \\
\text { St Pierre (10 perches) }\end{array}$ & $9-10$ & $5-6$ & $40-50$ pieds \\
\hline 4 & $\begin{array}{c}\text { Moulin St Pierre/pont St Pierre } \\
\text { (7-8 perches) }\end{array}$ & $5-6$ & 1 & $40-50$ pieds \\
\hline 5 & $\begin{array}{c}\text { Pont St Pierre/quai des } \\
\text { Carmes (150 perches) }\end{array}$ & $5-6$ & 1 & $40-50$ pieds \\
\hline 6 & $\begin{array}{c}\text { Quai des Carmes/quai du pont } \\
\text { de Vaucelles (15 perches) }\end{array}$ & $6-7$ & 1 & 60 pieds \\
\hline 7 & $\begin{array}{c}\text { Quai du pont de Vaucelles/ } \\
\text { "gambe d'aulne " (15 perches) }\end{array}$ & $11-12$ & 8 & - \\
\hline 8 & $\begin{array}{c}\text { Quais et muraille de Caen/bas } \\
\text { de Colombelles }\end{array}$ & $12-13$ & $7-8$ & 70 pieds \\
\hline 9 & $\begin{array}{c}\text { Colombelles/Bénouville } \\
\text { (une lieue) }\end{array}$ & $6-7$ & 1 & 6-8 perches \\
\hline
\end{tabular}

Source : SHD Vincennes, SH 48.

107. Dans le domaine économique, Caen est conçue avant tout comme un lieu d'échanges, dominé d'une part par le port capable d'accueillir des bateaux de 60 tx, et les foires, d'autre part (LEMIÈRE, Luc, "Discours des élites et réalités urbaines : l'exemple de Caen au XVII ${ }^{\mathrm{e}}$ siècle ", Cahier des Annales de Normandie, n 19, 1985, p. 290).

108. Dans la section du quai du pont de Vaucelles proche du lieu de chargement et déchargement des navires, cette section méandreuse de l'Orne divisée en deux parties « a esté emplye depuis trente ans [...] qui est la Cause que de basse eau Il ny a que la haulteur d'un pied deau et six a sept pieds de plaine mer de hauteur deau " (SHD Vincennes, SH 48). 
Édouard DelobetTe
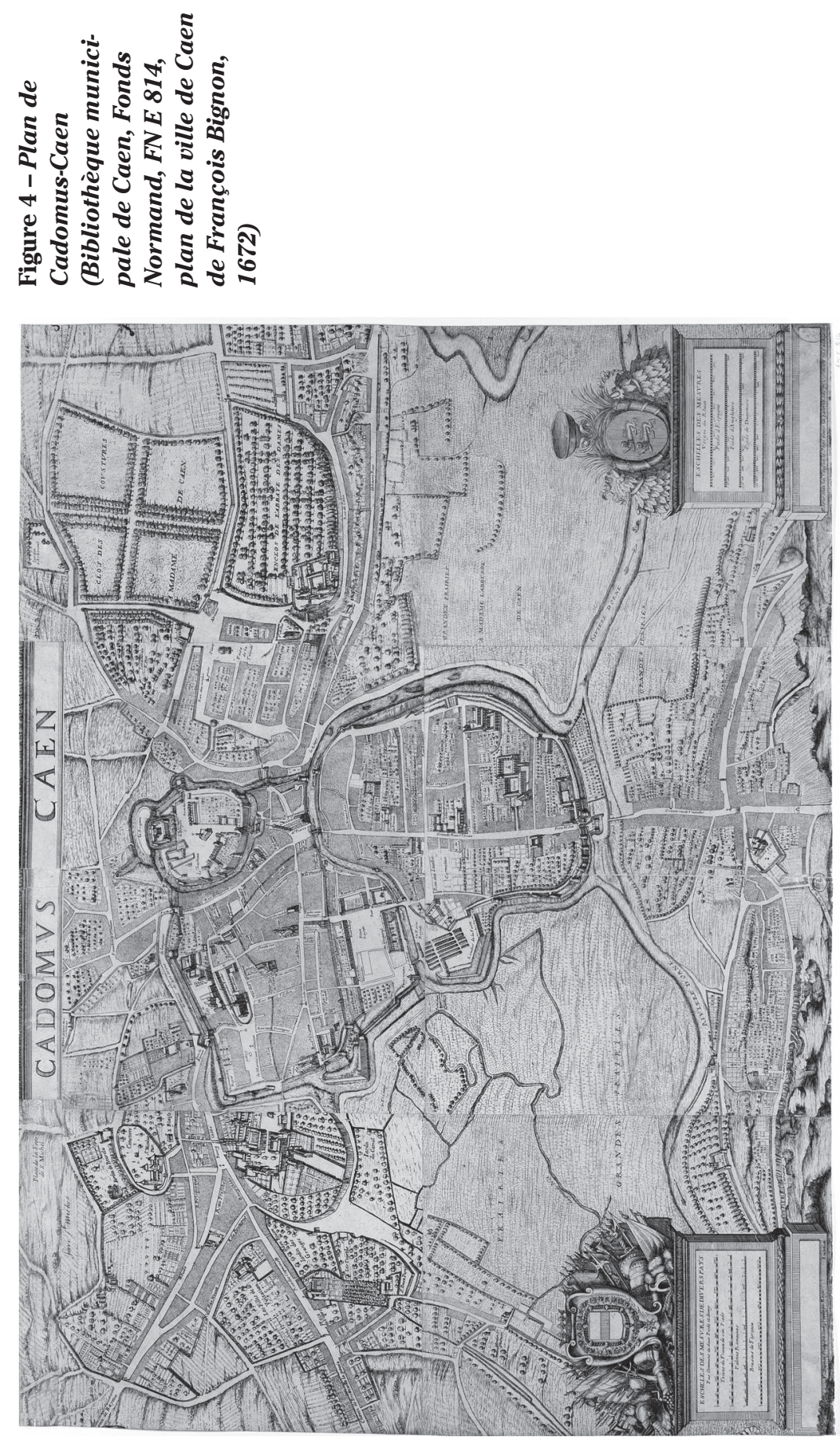
La faible profondeur de l'Orne constatée à marée basse de morte-eau dans les secteurs $n^{\circ} 4$ à 6 provient des nombreuses négligences de particuliers comme des communautés, sans y impliquer le lestage ou délestage des navires qui se produit à proximité d'une grosse tour, "sans apporter aucun dommage à la rivière ». Dans la section partant du moulin Saint-Pierre jusqu'au pont Saint-Pierre, la profondeur de l'Orne s'établit à seulement un pied d'eau " à cause des murs de la ville qui sont tombéz et tombent encor journellement depuis dix ans dans la rivière lesquelles pierres y sont encore de présent en la plus grande partye et les autres que les eaux auroient faict descendre aval la rivière le long des quays ${ }^{109}$ ». Du pont Saint-Pierre au quai des Carmes, la très faible profondeur est également due à l'éboulement des pierres et murs de la ville ainsi que des " tallutz " qui bordaient la rivière autrefois du côté du faubourg et paroisse Saint Gilles. À côté du pont SaintJacques vis-à vis la maison du sieur Mendrac, il reste depuis cinq ans, sur le bord de la rivière, provenant du " bastiement " de cette maison un gros monceau de pierres et de terre, dont une grande partie est déjà tombée dans la rivière.

Les conflits d'usage entre riverains et mariniers sont fréquents sur l'Orne. Les empiètements dans le lit de la rivière commis par les propriétaires riverains se sont multipliés. Ainsi, dans la section $\mathrm{n}^{\circ} 2$, près de la grosse tour située à proximité de la demeure du sieur La Perelle, la maison située vis-à-vis de celle du sieur de la Varende et construite il y a vingt-cinq ans par l'actuel propriétaire, le sieur de Manneville, trésorier de France à Caen, a ses fondations en partie avancées de 6-7 pieds dans la rivière; elle penche et menace de s'effondrer dans la rivière, risquant d'entraîner avec elle une partie du pont. Comme si cela ne suffisait pas, le siège de l'amirauté de Caen instruit contre une malversation portant sur le marché du curage de l'Orne. Les anciens et experts désignés confirment la somme de 500 livres allouée chaque année par les maire et échevins de Caen pour le curage de la rivière et des quais, bien que la rivière ne soit plus curée depuis plus de trente-cinq ans, la dernière adjudication ayant été remportée par un sieur Michel Fouquet. Les anciens dénoncent également les exactions et abus commis par le nommé Jean Troussel, soi-disant nommé par les échevins, et qui fait indûment payer aux marchands, maîtres et patrons de barque un droit de curage et de pierrage de la rivière, " ce quil a faict paier depuis longtemps et néantmoingts lesdicts eschevins ny ledict troussel non faict curer ladite riviere depuis l'adiudication dudict fouquet ${ }^{110}$ ".

109. La " crétine " ou crue et inondation de janvier 1651 a abattu les murailles de Caen situées le long de la Foire du pré (GARNIER, Emmanuel, " La ville face aux caprices du fleuve. L'exemple normand, $\mathrm{XVI}^{\mathrm{e}}$-XVIII ${ }^{\mathrm{e}}$ siècle ", Histoire urbaine, $\mathrm{n}^{\circ} 18,2007$, p. 50). Sur les accidents climatiques survenus à Rouen depuis 1496, voir FARIN, Histoire de la Ville de Roüen, Rouen, chez Jacques Amiot, sur le Quay, 1710, nlle éd., t. I, p. 385-et 391.

110. Information, audition et confrontation de témoins devant Hervey Fossey, lieutenant du siège, à la diligence du procureur du roi Pierre Collet au siège de la juridiction, le 12 mai 1664, contre Jean et Pierre Troussel père et fils, accusés d'avoir indûment procédé à des levées de deniers et exactions commises sur les maîtres de navires et marchands, 
Les rapports des lieutenants des sièges mentionnent en dernier lieu quelques préconisations des déposants afin de remédier rapidement aux dégradations les plus importantes. En Haute-Normandie, l'édification de jetées de bois ou de pierres perpendiculairement à l'estran semble la parade la plus couramment employée contre l'invasion du galet dans les ports, en dehors du complexe système hydraulique des chasses en usage dans de grands ports comme Le Havre. En Basse-Normandie, les anciens et experts de l'amirauté de Caen rappellent l'urgence de curer et " retaluter " la rivière le long des murailles de Caen, car " s'il n'y est promptement remédyé, ladicte riviere sera entièrement ruinée et ne pourra plus estre navigable ${ }^{111}$ ". Ils ajoutent la nécessité de faire des ponts et de poser des planches au sol le long du chemin de halage en plusieurs endroits afin de faciliter le " hallage et bordage ". Ils réclament enfin des mesures contre les usurpations des rives par les propriétaires riverains et bordiers de la rivière. En aval de Caen, les anciens pilotes de l'Orne conseillent la consolidation des rives par l'implantation de " digues, épicz, quays ou autre bordure en plusieurs endroitz de ladicte riviere ", de Colombelles vers Caen comme depuis Bénouville vers la mer. Dans la baie des Veys, la remontée des navires de 100 tx vers Carentan nécessite deux marées au lieu d'une en raison d'un large méandre après le Four de Taute qui allonge inutilement le parcours. Pour ne plus remonter qu'en une seule marée, les anciens et matelots du lieu suggèrent de rectifier le cours de la rivière par le creusement de deux canaux.

En conclusion, le SH 48 présente un grand intérêt, par la description détaillée dans les sièges d'amirauté, au moment même où Colbert met en œuvre son grand dessein maritime, de presque tous les ports et littoraux du royaume ainsi que leurs problématiques naturelles. La recherche d'un site naturel favorable à la construction d'un port de guerre en Normandie conduit les officiers des sièges d'amirauté à collecter de précieuses informations sur les caractéristiques des rades et havres de leur ressort (nature des fonds, amplitude des marées, profondeurs). L'analyse des procès-verbaux des sièges d'amirauté normands montre, avec parfois une grande richesse de détails, l'évolution du trait de côte soit par le retrait du littoral (érosion, effondrement, submersion), soit au contraire par surhaussement, comblement, envasement ou ensablement progressif des havres ou petits estuaires de la province. Les rivières navigables sujettes aux marées comme

lorsqu'un nommé Saint-Amand, sergent de la garnison du château de Caen, entre dans le siège de la juridiction le chapeau sur la tête et l'épée au côté en proférant ces termes : "Mort dieu messieurs de la marauté vous faictes Informer Contre troussel mais prenes bien garde, a ce que vous ferez Car mortdieu messieurs les maraults vous ne vous trouverez pas mieux vous scavez Comme Il vous en [prend] \& vous verrez Comme Il vous en prendra encor..." (SHD Vincennes, SH 48, Caen.)

111. Le problème de la navigation sur l'Orne subsiste toujours en 1750 (DARSEL, Joachim, "L'Amirauté en Normandie. XI. Amirauté de Caen ", Annales de Normandie, 29e année, $\mathrm{n}^{\circ} 1,1979$, p. 48). 
la Seine ou l'Orne font également l'objet d'une description de leur cours. Les procès-verbaux du SH 48 dressent avec précision l'état des lieux de l'outil portuaire normand. On peut en apprécier l'état de dégradation très avancée des infrastructures et interpréter les causes d'un tel délabrement car la guerre maritime en Manche contre l'Espagne ou l'Angleterre ne peut tout justifier à elle seule. La responsabilité de "l'État Mazarin " ainsi que les négligences des communautés portuaires et de leurs élites y sont aussi pour beaucoup.

L'enquête a-t-elle été d'une réelle utilité? Cette question appelle une réponse de Normand. Si l'historien maritimiste fait son miel de la masse d'informations contenues dans le $\mathrm{SH} 48$, les attentes de Colbert ont été fortement déçues dans la mesure où aucun siège d'amirauté de Normandie n'a répondu de manière exhaustive à son questionnaire détaillé. Au-delà des inerties locales des officiers d'amirauté, la raison principale est simple à comprendre : aucun site côtier de Normandie ne présente l'ensemble des caractéristiques naturelles propres à pouvoir abriter une flotte de guerre et d'en permettre l'accès en permanence aux navires de combat, comme le rappelle Jean Meyer ${ }^{112}$. En revanche, le SH 48 propose un éclairage supplémentaire pour comprendre la décision de Colbert d'agrandir Brest, de fonder Rochefort et de substituer Lorient au Havre pour y armer la plupart des vaisseaux de la Compagnie des Indes orientales. En dernier lieu, le SH 48 gagnerait aussi à être exploité dans le cadre d'autres provinces maritimes ${ }^{113}$. La mise en relation du SH 48 avec le célèbre " inventaire " de la flotte marchande de 1664 tracerait alors le paysage d'une magnifique et inédite perspective de la France maritime au début du règne personnel de Louis XIV ${ }^{114}$.

112. "Mais, au nord-est de Brest, il n'y a plus aucun port français utilisable par une escadre de ligne. On a trop rarement souligné le paradoxe voulu par la nature. La rive nord de la Manche : la côte anglaise, offre tout une série de rades foraines assez bien protégées, et de rades admirables comme celle de Southampton; la rive sud n'offre rien, ou pas grand-chose. Car il faut tenir compte des tirants d'eau. " (MEYER, Jean, Béveziers (1690). La France prend la maîtrise de la Manche, Paris, Economica, 1993, p. 25-26.)

113. Delobette, Édouard, "L'enquête des amirautés de 1665 en France à travers deux études de cas : le Bourg d'Ault et Saint-Valéry-sur-Somme ", Revue d'histoire maritime, à paraître dans la livraison du ${ }^{\circ} 19$ en 2014.

114. Bnf, Cinq Cents Colbert, $\mathrm{ms} \mathrm{n}^{\circ} 199$. 


\section{RÉSUMÉ}

La grande enquête des amirautés lancée au printemps 1665 par Colbert est une mine d'informations sur la situation des ports et havres du royaume au début du règne personnel de Louis XIV. L'exemple de la Normandie littorale souligne dans cette enquête le médiocre potentiel portuaire de la province, le délabrement général des installations portuaires et l'héritage pesant du dernier conflit maritime franco-espagnol.

La mise en ouvre portuaire et commerciale du " grand dessein " de Colbert en Normandie se heurte rapidement à ses limites techniques et financières tout en suscitant le mécontentement des milieux d'affaires, notamment dieppois, impliqués dans le grand commerce atlantique.

Tenus à l'écart du grand dessein de réhabilitation portuaire de Colbert, certains ports et havres de Basse-Normandie, parfois spécialisés dans l'approvisionnement alimentaire de Paris, sont l'objet de toutes les attentions des intendants qui veillent à les intégrer dans les circuits d'exportation des nouvelles manufactures établies dans la généralité de Caen.

\section{ABSTRACT}

The great administrative survey of all French admiralty courts launched by Colbert in the Spring of 1665 contains a wealth of information about the condition of ports and harbours of the French kingdom at the beginning of Louis XIV's personal reign. In Normandy, it was shown that the coastal area had poor development potential, the port installations had gone to ruin and the difficult legacy of the recent naval conflict with the Spanish.

When Colbert began elaborating his grand design to improve port facilities and trading in Normandy, he was hampered by technical and budget difficulties as well as growing discontent among businessmen in Upper Normandy (especially in Dieppe) who were involved in the Atlantic system.

Certain Lower Normandy ports and harbours, some of them deeply involved in the Parisian food supply chain, were excluded from this great restoration project, but were instead the focus of the King's intendants' attention as they sought to include them in the export channels of the new manufactories established in the Généralité de Caen. 